TRANSACTIONS OF THE

AMERICAN MATHEMATICAL SOCIETY

Volume 349, Number 6, June 1997, Pages 2125-2173

S $0002-9947(97) 01738-8$

\title{
RAMANUJAN'S CLASS INVARIANTS, KRONECKER'S LIMIT FORMULA, AND MODULAR EQUATIONS
}

\author{
BRUCE C. BERNDT, HENG HUAT CHAN, AND LIANG-CHENG ZHANG
}

In Memory of Jerry Keiper

\begin{abstract}
In his notebooks, Ramanujan gave the values of over 100 class invariants which he had calculated. Many had been previously calculated by Heinrich Weber, but approximately half of them had not been heretofore determined. G. N. Watson wrote several papers devoted to the calculation of class invariants, but his methods were not entirely rigorous. Up until the past few years, eighteen of Ramanujan's class invariants remained to be verified. Five were verified by the authors in a recent paper. For the remaining class invariants, in each case, the associated imaginary quadratic field has class number 8 , and moreover there are two classes per genus. The authors devised three methods to calculate these thirteen class invariants. The first depends upon Kronecker's limit formula, the second employs modular equations, and the third uses class field theory to make Watson's "empirical method"rigorous.
\end{abstract}

\section{INTRODUCTION}

So that we may define Ramanujan's class invariants, set

$$
(a ; q)_{\infty}=\prod_{n=0}^{\infty}\left(1-a q^{n}\right), \quad|q|<1,
$$

and

$$
\chi(q)=\left(-q ; q^{2}\right)_{\infty} .
$$

If $q=\exp (-\pi \sqrt{n})$, the two class invariants $G_{n}$ and $g_{n}$ are defined by

$$
G_{n}:=2^{-1 / 4} q^{-1 / 24} \chi(q) \quad \text { and } \quad g_{n}:=2^{-1 / 4} q^{-1 / 24} \chi(-q) .
$$

In the notation of Weber [36], $G_{n}=: 2^{-1 / 4} f(\sqrt{-n})$ and $g_{n}=: 2^{-1 / 4} f_{1}(\sqrt{-n})$. It is well-known that $G_{n}$ and $g_{n}$ are algebraic; for example, see Cox's book [11, p. 214, Theorem 10.23; p. 257, Theorem 12.17].

At scattered places in his first notebook [23], Ramanujan recorded the values for 107 class invariants. On pages 294-299 in his second notebook [23], Ramanujan gave a table of values for 77 class invariants, three of which are not found in the first notebook. Since the second notebook is an enlarged revision of the first, it is unclear why Ramanujan failed to record 33 class invariants that he offered in the

Received by the editors March 16, 1995 and, in revised form, November 14, 1995.

1991 Mathematics Subject Classification. Primary 11R29; Secondary 11R04, 11R37, 11R42, 11F27, 33D10. 
first notebook. By the time Ramanujan wrote his paper [22], [24, pp. 23-39], he was aware of Weber's work [36], and so his table of 46 class invariants in [22] does not contain any that are found in Weber's book [36]. Except for $G_{325}$ and $G_{363}$, all of the remaining values are found in Ramanujan's notebooks.

In two papers [30], [31], G. N. Watson proved 24 of Ramanujan's class invariants from [22]. In the first [30], Watson devised an "empirical process" to calculate 14 of the 24 invariants, while in the second [31], he employed modular equations to prove 10 invariants. In another paper [29], Watson established Ramanujan's value for $G_{1353}$, communicated by him in his first letter to Hardy [24, p. xxix], and also stated in [22]. Watson wrote four further papers [32]-[35] on the calculation of class invariants. Among the dozens of invariants calculated by Watson in these papers were three previously unproved invariants found in Ramanujan's paper [22], as well as 11 invariants of Ramanujan that had been previously verified. Thus, after Watson's work, 18 invariants of Ramanujan from his paper and notebooks [23] remained to be verified.

The authors established five of these invariants in [4]. For each of these five values, $n$ is a multiple of 9 , and proofs were effected by formulas relating $G_{9 n}$ with $G_{n}$ and $g_{9 n}$ with $g_{n}$. As a bonus, the latter two formulas led to closed form evaluations of Ramanujan's cubic continued fraction at the arguments $\pm \exp (-\pi \sqrt{n})$.

The purpose of this paper is to establish the remaining 13 values, each for $G_{n}, n=$ $65,69,77,141,145,205,213,217,265,301,445,505,553$, claimed by Ramanujan. Quite remarkably, the class number for each of the 13 imaginary quadratic fields $\mathbb{Q}(\sqrt{-n})$ equals 8. Moreover, there are precisely two classes per genus in each case. Our first proofs employ the Kronecker limit formula, which is used to find representations for certain products of Dedekind eta-functions in terms of fundamental units; see Theorems 3.1,3.2, and 5.2. Each of the 13 values of $n$ is a product of a small prime $(3,5$, or 7$)$ and a larger prime. Thus, our proofs, given in Sections $2-4$, also crucially employ certain modular equations of Ramanujan of degrees 3,5 , and 7. It is highly unlikely that Ramanujan was familiar with the Kronecker limit formula and the arithmetic of quadratic fields, and so our proofs certainly are not those found by Ramanujan. However, Ramanujan obviously discerned some unique arithmetical properties in these instances, and it would be fascinating to discover Ramanujan's approach.

To make clearer the connection between modular equations and class invariants, we first give a precise definition of a modular equation. Let $K, K^{\prime}, L$, and $L^{\prime}$ denote complete elliptic integrals of the first kind associated with the moduli $k$, $k^{\prime}:=\sqrt{1-k^{2}}, \ell$, and $\ell^{\prime}:=\sqrt{1-\ell^{2}}$, respectively, where $0<k, \ell<1$. Suppose that

$$
n \frac{K^{\prime}}{K}=\frac{L^{\prime}}{L}
$$

for some positive rational number $n$. If $n$ is a positive integer, a relation between $k$ and $\ell$ induced by (1.3) is called a modular equation of degree $n$. Following Ramanujan, set

$$
\alpha=k^{2} \quad \text { and } \quad \beta=\ell^{2} .
$$

We often say that $\beta$ has degree $n$ over $\alpha$. As usual, in the theory of elliptic functions, set

$$
q:=\exp \left(-\pi K^{\prime} / K\right)
$$


Note that if $K^{\prime} / K=\sqrt{n}$ (where $n$ is not necessarily the degree of the modular equation), then

$$
q=\exp (-\pi \sqrt{n})
$$

which is the value of $q$ in our definition (1.2) of $G_{n}$. Since $\chi(q)=$ $2^{1 / 6}\{\alpha(1-\alpha) q\}^{-1 / 24}[1$, p. 124], it follows from (1.1), (1.2), and (1.5) that

$$
G_{n}=\{4 \alpha(1-\alpha)\}^{-1 / 24}
$$

Lastly, we recall that if

$$
\varphi(q)=\sum_{n=-\infty}^{\infty} q^{n^{2}}, \quad|q|<1,
$$

then $[1$, p. 102]

$$
\varphi(q)=\frac{\pi}{2} K(\sqrt{\alpha})
$$

when $q$ is given by (1.4).

Ramanujan used modular equations to calculate only a couple of simple invariants in [22]. This fact and the sentence, "The values of $G_{n}$ and $g_{2 n}$ are got from the same modular equation." [22], [24, p. 25] are the only clues to his methods that Ramanujan provided for us. It would seem that if Ramanujan had employed another type of reasoning, he would have dropped some hint about it. As mentioned earlier, Watson [31] used modular equations to establish some of Ramanujan's invariants. However, for his calculations of $G_{n}$, it was important that $n$ be a square or a simple multiple of a square. We have been able to prove six of the thirteen values for $G_{n}$, namely, for $n=65,69,77,141,145,213$, by using modular equations. As will be seen in our proofs in Section 6, we need some new ideas to effect proofs of these six invariants via modular equations. To prove the remaining seven invariants by employing modular equations, we would need modular equations of degrees 31,41 , 43, 53, 79, 89, and 101. Apparently, only for degree 31 did Ramanujan derive a modular equation, for he recorded no modular equations for the other six degrees in his notebooks. Thus, Ramanujan's methods appear to be even more elusive.

Watson [30, p. 82] opined that "I believe that fourteen were obtained by Ramanujan by means of the empirical process which I described in the discussion of $G_{1353 .}$. As indicated in our paper [4], we believe that Ramanujan found some of these values by the method of [4]. We are not so confident that Ramanujan used this empirical process, for which Watson offered little explanation. In fact, Watson's "empirical process" is not rigorous. However, in Section 7 we shall use class field theory to make Watson's procedure rigorous for a large class of invariants including those 13 invariants examined in this paper, and we use the process to calculate two new invariants as well.

\section{Kronecker's Limit Formula and Background}

Let $Q(u, v):=y^{-1}(u+v z)(u+v \bar{z})$, where $z=x+i y$ with $y>0$. The Epstein zeta-function $\zeta_{Q}(s)$ is defined for $\sigma=\operatorname{Re} s>1$ by

$$
\zeta_{Q}(s):=\sum_{u, v}\{Q(u, v)\}^{-s}
$$

where the sum is over all pairs of integers $(u, v)$ except $(0,0)$. It is well known that $\zeta_{Q}(s)$ can be analytically continued to the entire complex $s$-plane, where $\zeta_{Q}(s)$ is 
analytic except for a simple pole at $s=1$. The Kronecker limit formula provides the constant term in the Laurent expansion about $s=1$. More precisely,

$$
\zeta_{Q}(s)=\frac{\pi}{s-1}+2 \pi\left(\gamma-\log 2-\log \left(\sqrt{y}|\eta(z)|^{2}\right)\right)+O(s-1),
$$

where $\gamma$ denotes Euler's constant, and $\eta(z)$ is the Dedekind eta-function defined by

$$
\eta(z):=q^{1 / 24}(q ; q)_{\infty}=: q^{1 / 24} f(-q), \quad q=e^{2 \pi i z}, y>0,
$$

where the notation $f(-q)$ is that used by Ramanujan in his notebooks [23].

Next, let $K$ be an algebraic number field over the rational numbers. Let $N(\mathfrak{A})$ denote the norm of an ideal $\mathfrak{A}$. Then the Dedekind zeta-function for $K$ is defined by

$$
\zeta_{K}(s):=\sum_{\mathfrak{A}}(N(\mathfrak{A}))^{-s}, \quad \sigma>1,
$$

where the sum is over all non-zero integral ideals $\mathfrak{A}$ of $K$. Let $C_{K}$ denote the ideal class group of $K$. Then the Dedekind zeta-function for an ideal class $A$ of $C_{K}$ is defined by

$$
\zeta(s, A):=\sum_{\mathfrak{A} \in A}(N(\mathfrak{A}))^{-s}, \quad \sigma>1 .
$$

If $\chi$ denotes an ideal class character, then the $L$-series for $K$ is given, for $\sigma>1$, by

$$
L_{K}(s, \chi):=\sum_{\mathfrak{A}} \chi(\mathfrak{A})(N(\mathfrak{A}))^{-s}=\sum_{A} \chi(A) \zeta(s, A),
$$

where the former sum is over all non-zero integral ideals $\mathfrak{A}$ of $K$, and the latter sum is over all ideal classes $A$ of $C_{K}$.

In the sequel we assume that $K$ is a quadratic field. It is well known that [27, p. 58]

$$
\lim _{s \rightarrow 1}(s-1) \zeta_{K}(s)=h \kappa,
$$

where $h$ is the class number of $K$, i.e., $h=\left|C_{K}\right|$, and where

$$
\kappa:= \begin{cases}\frac{2 \pi}{w \sqrt{-d}}, & \text { if } K \text { is imaginary } \\ \frac{2 \log \epsilon}{\sqrt{d}}, & \text { if } K \text { is real. }\end{cases}
$$

Here $w$ is the number of roots of unity in $K, d$ is the discriminant of $K$, and $\epsilon$ is the fundamental unit in $K$.

Let

$$
L_{d}(s):=\sum_{n=1}^{\infty}\left(\frac{d}{n}\right) n^{-s}, \quad \sigma>1,
$$

where $\left(\frac{d}{n}\right)$ is the Kronecker symbol. Then [27, p. 58]

$$
\zeta_{K}(s)=\zeta(s) L_{d}(s)
$$

where $\zeta(s)$ denotes the Riemann zeta-function. 
Now let $d=d_{1} d_{2}$, where $d_{1}>1$ and, for $i=1,2, d_{i} \equiv 1(\bmod 4)$ or $d_{i} \equiv 0$ $(\bmod 4)$. Let $\mathfrak{P}$ denote a prime ideal in $K$. Then a Gauss genus character $\chi$ is defined by

$$
\chi(\mathfrak{P})= \begin{cases}\left(\frac{d_{1}}{N(\mathfrak{P})}\right), & \text { if } N(\mathfrak{P}) \nmid d_{1}, \\ \left(\frac{d_{2}}{N(\mathfrak{P})}\right), & \text { if } N(\mathfrak{P}) \mid d_{1},\end{cases}
$$

where $\left(\frac{d_{i}}{N(\mathfrak{P})}\right)$ again denotes the Kronecker symbol. Note that $N(\mathfrak{P}) \nmid d_{2}$ if $N(\mathfrak{P}) \mid d_{1}$. This definition can be extended to all ideals of $K$ by multiplicativity. It is well known that the genus characters form an abelian group, denoted by $G(K)$, of order $2^{k-1}$, where $k$ is the number of distinct prime divisors of $d$.

Next define

$$
\boldsymbol{G}_{0}:=\left\{A \in C_{K}: \chi(A)=1, \chi \in G(K)\right\},
$$

which is named the principal genus. Clearly, $\boldsymbol{G}_{0}$ is a subgroup of $C_{K}$, and $C_{K} / \boldsymbol{G}_{0}$ is called the genus group. Furthermore, $C_{K} / \boldsymbol{G}_{0} \cong G(K)$. Obviously, $A_{1}$ and $A_{2}$ are in the same genus if and only if $\chi\left(A_{1}\right)=\chi\left(A_{2}\right)$ for each $\chi \in G(K)$.

Kronecker $[27$, p. 62 , Theorem 4] proved that, for a genus character $\chi$ of $K$ corresponding to the decomposition $d=d_{1} d_{2}$,

$$
L_{K}(s, \chi)=L_{d_{1}}(s) L_{d_{2}}(s) .
$$

Thus, by (2.4) and (2.8),

$$
L_{d_{1}}(s) L_{d_{2}}(s)=\sum_{A \in C_{K}} \chi(A) \zeta(s, A) .
$$

For a fixed non-zero integral ideal $\mathfrak{B} \in A^{-1}$,

$$
\zeta(s, A)=N(\mathfrak{B})^{s} \sum_{\mathfrak{A} \in A}(N(\mathfrak{A} \mathfrak{B}))^{-s}=N(\mathfrak{B})^{s} \sum_{\lambda \in \mathfrak{B} / U}(N(\lambda))^{-s}, \quad \sigma>1,
$$

where $U$ is the group of units in $K$. Now assume that $K=\mathbb{Q}(\sqrt{-m})$ is an imaginary quadratic field, and so $m$ is a squarefree positive integer. Recalling that $w$ is the number of roots of unity in $K$, we see that, from (2.9),

$$
\zeta(s, A)=\frac{N(\mathfrak{B})^{s}}{w} \sum_{\substack{\lambda \in \mathfrak{B} \\ \lambda \neq 0}}(N(\lambda))^{-s}, \quad \sigma>1 .
$$

Let

$$
\Omega= \begin{cases}\sqrt{-m}, & \text { if }-m \equiv 2,3 \quad(\bmod 4), \\ (1+\sqrt{-m}) / 2, & \text { if }-m \equiv 1 \quad(\bmod 4) .\end{cases}
$$

Then

$$
d=\left\{\begin{aligned}
-4 m, & \text { if }-m \equiv 2,3 \quad(\bmod 4), \\
-m, & \text { if }-m \equiv 1 \quad(\bmod 4)
\end{aligned}\right.
$$

It is known [15] that each ideal class contains primitive ideals which are $\mathbb{Z}$-modules of the form $\mathfrak{B}=[a, b+\Omega]$, where $a$ and $b$ are rational integers, $a>0, a|N(b+\Omega)| b \mid, \leq$ $a / 2, a$ is the smallest positive integer in $\mathfrak{B}$, and $N(\mathfrak{B})=a$. 
Let $z=(b+\Omega) / a$. Then, for $\lambda=u a+v(b+\Omega)$,

$$
\begin{aligned}
N(\lambda) & =(u a+v(b+\Omega))(u a+v(b+\bar{\Omega})) \\
& =a^{2}(u+v z)(u+v \bar{z}) \\
& =\frac{a \sqrt{|d|}}{2}\left(\frac{\sqrt{|d|}}{2 a}\right)^{-1}(u+v z)(u+v \bar{z}) .
\end{aligned}
$$

Thus, for $z=(b+\Omega) / a$ and $y=\operatorname{Im} z=\sqrt{|d|} /(2 a)$,

$$
Q(u, v)=\left(\frac{\sqrt{|d|}}{2 a}\right)^{-1}(u+v z)(u+v \bar{z}) .
$$

And, from (2.1), (2.10), and (2.11),

$$
\zeta(s, A)=\frac{1}{w}\left(\frac{2}{\sqrt{|d|}}\right)^{s} \zeta_{Q}(s) .
$$

Thus, from (2.2),

$$
\begin{aligned}
\zeta(s, A)= & \frac{1}{w}\left(\frac{2}{\sqrt{|d|}}\right)^{s}\left(\frac{\pi}{s-1}+2 \pi \gamma-2 \pi \log 2+\frac{\pi}{2} \log \sqrt{|d|}\right) \\
& -\frac{2 \pi}{w}\left(\frac{2}{\sqrt{|d|}}\right)^{s}\left(-\frac{1}{2} \log (2 a)+\log |\eta(z)|^{2}\right)+O(s-1) .
\end{aligned}
$$

Since, for any nonprincipal genus character $\chi$,

$$
\sum_{A \in C_{K}} \chi(A)=0,
$$

it follows from (2.4) and (2.12) that

$$
L_{K}(s, \chi)=-\frac{2 \pi}{w}\left(\frac{2}{\sqrt{|d|}}\right)^{s} \sum_{A \in C_{K}} \chi(A)\left(-\frac{1}{2} \log a+\log |\eta(z)|^{2}\right)+O(s-1) .
$$

Recall that in the decomposition $d=d_{1} d_{2}$ we assume that $d_{1}>1$ and $d_{2}<0$. Let $K_{i}=\mathbb{Q}\left(\sqrt{d_{i}}\right), i=1,2$. By $(2.7)$,

$$
\lim _{s \rightarrow 1}(s-1) \zeta_{K_{i}}(s)=L_{d_{i}}(1), \quad i=1,2 .
$$

Then, by (2.5) and (2.6),

$$
L_{d_{1}}(1)=\frac{2 h_{1} \log \epsilon_{1}}{\sqrt{d_{1}}}
$$

and

$$
L_{d_{2}}(1)=\frac{2 h_{2} \pi}{w_{2} \sqrt{\left|d_{2}\right|}},
$$

where $h_{i}$ is the class number of $K_{i}, i=1,2, \epsilon_{1}$ is the fundamental unit of $K_{1}$, and $w_{2}$ is the number of roots of unity in $K_{2}$. Thus, setting $s=1$ in (2.13) and using 
(2.8), we deduce that

$$
L_{d_{1}}(1) L_{d_{2}}(1)=-\frac{4 \pi}{w \sqrt{|d|}} \sum_{A \in C_{K}} \chi(A)\left(-\frac{1}{2} \log a+\log |\eta(z)|^{2}\right) .
$$

Thus, setting

$$
F(A)=|\eta(z)|^{2} / \sqrt{a},
$$

where $z=(b+\Omega) / a$, with $[a, b+\Omega] \in A^{-1}$, we conclude from (2.14)-(2.17) that, for $\chi$ nonprincipal $[27$, p. 72$]$,

$$
\frac{w h_{1} h_{2} \log \epsilon_{1}}{w_{2}}=-\sum_{A \in C_{K}} \chi(A) \log F(A),
$$

or

$$
\epsilon_{1}^{w h_{1} h_{2} / w_{2}}=\prod_{A \in C_{K}} F(A)^{-\chi(A)}
$$

We remark that (2.18) was utilized by K. G. Ramanathan [17], [18], [19], [20], [21] to calculate class invariants, values of the Rogers-Ramanujan continued fraction, and certain other invariants of Ramanujan.

\section{Two Primary Theorems}

Let $\tau=\sqrt{-m}$. Then, by (1.1) and (1.2), it is easily seen that

$$
\left|\frac{\eta\left(\frac{\tau+1}{2}\right)}{\eta(\tau)}\right|=2^{1 / 4} G_{m} .
$$

Equalities (2.18) and (3.1) are the key ingredients for deriving formulas that will enable us to calculate $G_{m}$. In this section, we consider two different genus structures, and the two theorems that we prove can be utilized to determine $G_{m}$ for $m=65$, $69,77,141,145,205,213,265,301,445,505$. For $m=217,553$, the genus structure is of a third type, and this type will be examined in Section 5. In each case, $K=\mathbb{Q}(\sqrt{-m})$ has class number 8 , and the number of genera equals 4 . Thus, each genus contains exactly two ideal classes. Also note that $A$ and $A^{-1}$ are clearly in the same genus.

Throughout this paper, for simplicity, we use the notation for a primitive ideal to denote the ideal class containing it; this abuse of notation should not cause difficulty.

Theorem 3.1. Let $m \equiv 1(\bmod 4)$, where $m$ is a positive squarefree integer with prime divisor $p$. Let $K=\mathbb{Q}(\sqrt{-m})$ be an imaginary quadratic field such that each genus contains exactly two ideal classes and such that the principal genus $\boldsymbol{G}_{0}$ contains the classes $[1, \Omega]$ and $[2 p, p+\Omega]$. Let $\boldsymbol{G}_{1}$ be a nonprincipal genus containing the two classes $[2,1+\Omega]$ and $[p, \Omega]$. Then

$$
\left(\frac{G_{m}}{G_{m / p^{2}}}\right)^{h / 2}=\prod_{\chi\left(\boldsymbol{G}_{1}\right)=-1} \epsilon_{1}^{w h_{1} h_{2} / w_{2}},
$$

where $h, h_{1}$, and $h_{2}$ are the class numbers of $K, \mathbb{Q}\left(\sqrt{d_{1}}\right)$, and $\mathbb{Q}\left(\sqrt{d_{2}}\right)$, respectively, $w$ and $w_{2}$ are the numbers of roots of unity in $K$ and $\mathbb{Q}\left(\sqrt{d_{2}}\right)$, respectively, $\epsilon_{1}$ is the 
fundamental unit in $\mathbb{Q}\left(\sqrt{d_{1}}\right)$, and the product is over all characters $\chi$ (with $\chi\left(\boldsymbol{G}_{1}\right)=$ $-1)$, associated with the decomposition $d=d_{1} d_{2}$, and therefore $d_{1}, d_{2}, h_{1}, h_{2}, w_{2}$, and $\epsilon_{1}$ are dependent on $\chi$.

Proof. Each of the ideals $[1, \Omega],[2 p, p+\Omega],[2,1+\Omega]$, and $[p, \Omega]$ is ambiguous. If $\mathfrak{A} \in A$ is any one of these ideals, then $\mathfrak{A} \sim \mathfrak{A}^{-1}, A=A^{-1}$, and $\mathfrak{A} \in A^{-1}$.

For any ideal class $B \notin \boldsymbol{G}_{0} \cup \boldsymbol{G}_{1}$, it is not difficult to see that [20, p. 77]

$$
\sum_{\chi\left(\boldsymbol{G}_{1}\right)=-1} \chi(B)=0
$$

which implies that

$$
\prod_{\chi\left(\boldsymbol{G}_{1}\right)=-1} F(B)^{-\chi(B)}=1,
$$

where $F(B)$ is defined by (2.17). Therefore, by (2.18),

$$
\prod_{\chi\left(\boldsymbol{G}_{1}\right)=-1} \epsilon_{1}^{w h_{1} h_{2} / w_{2}}=\prod_{\chi\left(\boldsymbol{G}_{1}\right)=-1} \prod_{A \in \boldsymbol{G}_{0} \cup \boldsymbol{G}_{1}} F(A)^{-\chi(A)}=\prod_{A \in \boldsymbol{G}_{0} \cup \boldsymbol{G}_{1}} F(A)^{-\chi(A) h / 4},
$$

since the number of genus characters equals $h / 2$, and so the number of genus characters with $\chi\left(\boldsymbol{G}_{1}\right)=-1$ is $h / 4$.

Let $A_{0}=[1, \Omega], A_{0}^{\prime}=[2 p, p+\Omega], A_{1}=[2,1+\Omega]$, and $A_{1}^{\prime}=[p, \Omega]$. Then, by $(3.2)$,

$$
\prod_{\chi\left(\boldsymbol{G}_{1}\right)=-1} \epsilon_{1}^{w h_{1} h_{2} / w_{2}}=\left(\frac{F\left(A_{1}\right) / F\left(A_{0}\right)}{F\left(A_{0}^{\prime}\right) / F\left(A_{1}^{\prime}\right)}\right)^{h / 4} .
$$

By (2.17) and (3.1),

$$
\frac{F\left(A_{1}\right)}{F\left(A_{0}\right)}=\frac{\eta^{2}\left(\frac{\Omega+1}{2}\right) / \sqrt{2}}{\eta^{2}(\Omega)}=G_{m}^{2}
$$

Let $\Omega^{\prime}=\Omega / p=\sqrt{-m / p^{2}}$. Again, by (2.17) and (3.1),

$$
\frac{F\left(A_{0}^{\prime}\right)}{F\left(A_{1}^{\prime}\right)}=\frac{\eta^{2}\left(\frac{\Omega+p}{2 p}\right) / \sqrt{2 p}}{\eta^{2}\left(\frac{\Omega}{p}\right)}=\frac{\eta^{2}\left(\frac{\Omega^{\prime}+1}{2}\right) / \sqrt{2}}{\eta^{2}\left(\Omega^{\prime}\right)}=G_{m / p^{2}}^{2} .
$$

The theorem now follows from (3.3)-(3.5).

Theorem 3.2. Let $m \equiv 1(\bmod 4)$, where $m$ is a positive squarefree integer with prime divisor $p$. Let $K=\mathbb{Q}(\sqrt{-m})$ be an imaginary quadratic field such that each genus contains exactly two ideal classes and such that the principal genus $\boldsymbol{G}_{0}$ contains the classes $[1, \Omega]$ and $[p, \Omega]$. Let $\boldsymbol{G}_{1}$ be a nonprincipal genus containing the two classes $[2,1+\Omega]$ and $[2 p, p+\Omega]$. Then

$$
\left(G_{m} G_{m / p^{2}}\right)^{h / 2}=\prod_{\chi\left(\boldsymbol{G}_{1}\right)=-1} \epsilon_{1}^{w h_{1} h_{2} / w_{2}},
$$

where $h, h_{1}$, and $h_{2}$ are the class numbers of $K, \mathbb{Q}\left(\sqrt{d_{1}}\right)$, and $\mathbb{Q}\left(\sqrt{d_{2}}\right)$, respectively, $w$ and $w_{2}$ are the numbers of roots of unity in $K$ and $\mathbb{Q}\left(\sqrt{d_{2}}\right)$, respectively, $\epsilon_{1}$ is the fundamental unit in $\mathbb{Q}\left(\sqrt{d_{1}}\right)$, and the product is over all characters $\chi$ (with $\chi\left(\boldsymbol{G}_{1}\right)=$ $-1)$, associated with the decomposition $d=d_{1} d_{2}$, and therefore $d_{1}, d_{2}, h_{1}, h_{2}, w_{2}$, and $\epsilon_{1}$ are dependent on $\chi$. 
The proof of Theorem 3.2 is analogous to that for Theorem 3.1, and so we omit it.

We say that $m$ is of the first kind or second kind according as it satisfies the conditions of Theorem 3.1 or Theorem 3.2, respectively.

It is not difficult to show that $[1, \Omega],[2,1+\Omega],[p, \Omega]$, and $[2 p, p+\Omega]$ are representatives of different ideal classes [15].

Before commencing our calculations we need three modular equations of Ramanujan [1, pp. 231, 282, 315].

Lemma 3.3 (Modular Equation of Degree 3). Let

$$
P=\{16 \alpha \beta(1-\alpha)(1-\beta)\}^{1 / 8} \quad \text { and } \quad Q=\left(\frac{\beta(1-\beta)}{\alpha(1-\alpha)}\right)^{1 / 4}
$$

Then

$$
Q+\frac{1}{Q}+2 \sqrt{2}\left(P-\frac{1}{P}\right)=0 \text {. }
$$

Lemma 3.4 (Modular Equation of Degree 5). Let

$$
P=\{16 \alpha \beta(1-\alpha)(1-\beta)\}^{1 / 12} \quad \text { and } \quad Q=\left(\frac{\beta(1-\beta)}{\alpha(1-\alpha)}\right)^{1 / 8}
$$

Then

$$
Q+\frac{1}{Q}+2\left(P-\frac{1}{P}\right)=0
$$

Lemma 3.5 (Modular Equation of Degree 7). Let

$$
P=\{16 \alpha \beta(1-\alpha)(1-\beta)\}^{1 / 8} \quad \text { and } \quad Q=\left(\frac{\beta(1-\beta)}{\alpha(1-\alpha)}\right)^{1 / 6}
$$

Then

$$
Q+\frac{1}{Q}+7=2 \sqrt{2}\left(P+\frac{1}{P}\right)
$$

Let $q=\exp (-\pi / \sqrt{n})$. Since $G_{n}=G_{1 / n}[22],\left[24\right.$, p. 23], by $(1.6), G_{n}=$ $\{4 \alpha(1-\alpha)\}^{-1 / 24}$. If $\beta$ has degree $p$ over $\alpha$, then $G_{n / p^{2}}=G_{p^{2} / n}=$ $\{4 \beta(1-\beta)\}^{-1 / 24}$. In summary, we can express the equalities of Lemmas 3.3-3.5 in terms of $G_{n}$ and $G_{n / p^{2}}, p=3,5,7$, respectively, by employing the formulas

$$
G_{n}=\{4 \alpha(1-\alpha)\}^{-1 / 24} \quad \text { and } \quad G_{n / p^{2}}=\{4 \beta(1-\beta)\}^{-1 / 24} .
$$

The class numbers cited below for $|d|<500$ can be found in tables in the texts of Borevich and Shafarevich [6, pp. 422-426], H. Cohen [10, pp. 503-509], and for $0<d<10,000$ in the book of D.A. Buell [8, pp. 224-234]. Lists of fundamental units can be found in [6] (for $d \leq 101$ ), the book by M. Pohst and H. Zassenhaus [16, pp. 432-435] (up to $d \leq 299$ ), and the tables of R. Kortum and G. McNiel [14] (up to $d=10,000$ ). In Cohen's book [10, pp. 262-274], there is a table providing the ideal class structure for $\mathbb{Q}(\sqrt{-d}), d \leq 97$ and for $\mathbb{Q}(\sqrt{d}), d \leq 97$. 


\section{Calculations of 11 Class Invariants}

Theorem 4.1.

$$
G_{65}=\left(\frac{\sqrt{13}+3}{2}\right)^{1 / 4}\left(\frac{\sqrt{5}+1}{2}\right)^{1 / 4}\left(\sqrt{\frac{9+\sqrt{65}}{8}}+\sqrt{\frac{1+\sqrt{65}}{8}}\right)^{1 / 2} .
$$

Proof. The following table summarizes the needed information about ideal classes and their characters.

\begin{tabular}{|c|c|c|c|c|c|c|c|c|c|c|}
\hline$d_{1}$ & $d_{2}$ & $\chi$ & $\boldsymbol{G}$ & $C$ & $\begin{array}{l}\chi\left(\boldsymbol{G}_{0}\right) \\
\chi\left(\boldsymbol{G}_{2}\right)\end{array}$ & $\begin{array}{l}\chi\left(\boldsymbol{G}_{1}\right) \\
\chi\left(\boldsymbol{G}_{3}\right)\end{array}$ & $h_{1}$ & $h_{2}$ & $w_{2}$ & $\epsilon_{1}$ \\
\hline 1 & -260 & $\chi_{0}$ & $\boldsymbol{G}_{0}$ & $\begin{array}{c}1, \Omega] \\
{[10,5+\Omega]}\end{array}$ & $\begin{array}{l}1 \\
1\end{array}$ & $\begin{array}{l}1 \\
1\end{array}$ & & & & \\
\hline 5 & -52 & $\chi_{1}$ & $\boldsymbol{G}_{1}$ & $\begin{array}{c}{[2,1+\Omega]} \\
{[5, \Omega]}\end{array}$ & $\begin{array}{r}1 \\
-1 \\
\end{array}$ & $\begin{array}{r}-1 \\
1 \\
\end{array}$ & 1 & 2 & 2 & $\frac{\sqrt{5}+1}{2}$ \\
\hline 13 & -20 & $\chi_{2}$ & $\boldsymbol{G}_{2}$ & $\begin{array}{c}{[3,1+\Omega]} \\
{[3,-1+\Omega]}\end{array}$ & $\begin{array}{l}1 \\
1\end{array}$ & $\begin{array}{l}-1 \\
-1\end{array}$ & 1 & 2 & 2 & $\frac{\sqrt{13}+3}{2}$ \\
\hline 65 & -4 & $\chi_{3}$ & $\boldsymbol{G}_{3}$ & $\begin{array}{c}{[6,1+\Omega]} \\
{[6,-1+\Omega]}\end{array}$ & $\begin{array}{r}1 \\
-1\end{array}$ & $\begin{array}{r}1 \\
-1\end{array}$ & & & & \\
\hline
\end{tabular}

Note that 65 is of the first kind. Applying Theorem 3.1 with $h=8$ and $w=2$, we find that

$$
\left(\frac{G_{65}}{G_{13 / 5}}\right)^{4}=\left(\frac{\sqrt{5}+1}{2}\right)^{2}\left(\frac{\sqrt{13}+3}{2}\right)^{2} .
$$

Let $Q=\left(G_{65} / G_{13 / 5}\right)^{3}$ and $P=\left(G_{65} G_{13 / 5}\right)^{-2}$. Then, by (4.1),

$$
Q=\left(\frac{\sqrt{5}+1}{2}\right)^{3 / 2}\left(\frac{\sqrt{13}+3}{2}\right)^{3 / 2}=(\sqrt{5}+2)^{1 / 2}(5 \sqrt{13}+18)^{1 / 2} .
$$

By Lemma 3.4,

$$
P^{-1}=\frac{\left(Q+Q^{-1}\right)+\sqrt{\left(Q+Q^{-1}\right)^{2}+16}}{4} .
$$

Now, by (4.2),

$$
\begin{aligned}
\left(Q+Q^{-1}\right)^{2}+16 & =Q^{2}+Q^{-2}+18 \\
& =(\sqrt{5}+2)(5 \sqrt{13}+18)+(\sqrt{5}-2)(5 \sqrt{13}-18)+18 \\
& =(5+\sqrt{65})^{2},
\end{aligned}
$$

and, by (4.4),

$$
Q+Q^{-1}=\sqrt{74+10 \sqrt{65}}
$$

Thus, by (4.3) and (4.4),

$$
P^{-1}=\frac{1}{4} \sqrt{74+10 \sqrt{65}}+\frac{1}{4}(5+\sqrt{65}) .
$$


Thus, by (4.2) and (4.6),

$$
\begin{aligned}
G_{65} & =Q^{1 / 6} P^{-1 / 4} \\
& =\left(\frac{\sqrt{5}+1}{2}\right)^{1 / 4}\left(\frac{\sqrt{13}+3}{2}\right)^{1 / 4}\left(\frac{1}{4} \sqrt{74+10 \sqrt{65}}+\frac{1}{4}(5+\sqrt{65})\right)^{1 / 4} .
\end{aligned}
$$

Thus, it remains to show that

$$
\frac{1}{4} \sqrt{74+10 \sqrt{65}}+\frac{1}{4}(5+\sqrt{65})=\left(\sqrt{\frac{9+\sqrt{65}}{8}}+\sqrt{\frac{1+\sqrt{65}}{8}}\right)^{2},
$$

which is easily shown by a routine calculation.

\section{Theorem 4.2.}

\begin{tabular}{|c|c|c|c|c|c|c|c|c|c|c|}
\hline$d_{1}$ & $d_{2}$ & $\chi$ & $\boldsymbol{G}$ & $C$ & $\begin{array}{l}\chi\left(\boldsymbol{G}_{0}\right) \\
\chi\left(\boldsymbol{G}_{2}\right)\end{array}$ & $\begin{array}{l}\chi\left(\boldsymbol{G}_{1}\right) \\
\chi\left(\boldsymbol{G}_{3}\right)\end{array}$ & $h_{1}$ & $h_{2}$ & $w_{2}$ & $\epsilon_{1}$ \\
\hline 1 & -276 & $\chi_{0}$ & $\boldsymbol{G}_{0}$ & $\begin{array}{c}{[1, \Omega]} \\
{[6,3+\Omega]}\end{array}$ & $\begin{array}{l}1 \\
1\end{array}$ & $\begin{array}{l}1 \\
1\end{array}$ & & & & \\
\hline 92 & -3 & $\chi_{1}$ & $\boldsymbol{G}_{1}$ & $\begin{array}{c}{[2,1+\Omega]} \\
{[3, \Omega]}\end{array}$ & $\begin{array}{l}1 \\
1\end{array}$ & $\begin{array}{l}-1 \\
-1 \\
\end{array}$ & 1 & 1 & 6 & $-5 \sqrt{23}$ \\
\hline 69 & -4 & $\chi_{2}$ & $\boldsymbol{G}_{2}$ & $\begin{array}{c}{[5,1+\Omega]} \\
{[5,-1+\Omega]}\end{array}$ & $\begin{array}{r}1 \\
-1\end{array}$ & $\begin{array}{r}-1 \\
1\end{array}$ & 1 & 1 & 4 & $\frac{25+3 \sqrt{69}}{2}$ \\
\hline 12 & -23 & $\chi_{3}$ & $\boldsymbol{G}_{3}$ & $\begin{array}{c}{[7,1+\Omega]} \\
{[7,-1+\Omega]}\end{array}$ & $\begin{array}{r}1 \\
-1\end{array}$ & $\begin{array}{r}1 \\
-1\end{array}$ & & & & \\
\hline
\end{tabular}

$$
G_{69}=\left(\frac{5+\sqrt{23}}{\sqrt{2}}\right)^{1 / 12}\left(\frac{3 \sqrt{3}+\sqrt{23}}{2}\right)^{1 / 8}\left(\sqrt{\frac{6+3 \sqrt{3}}{4}}+\sqrt{\frac{2+3 \sqrt{3}}{4}}\right)^{1 / 2} .
$$

Proof. We summarize the needed information in the following table.

We apply Theorem 3.1 with $h=8$ and $w=2$, as 69 is of the first kind. Thus,

$$
\left(\frac{G_{69}}{G_{23 / 3}}\right)^{4}=(24+5 \sqrt{23})^{1 / 3}\left(\frac{25+3 \sqrt{69}}{2}\right)^{1 / 2} .
$$

Let $Q=\left(G_{69} / G_{23 / 3}\right)^{6}$ and $P=\left(G_{69} G_{23 / 3}\right)^{-3}$. By $(4.7)$,

$$
\begin{aligned}
Q & =(24+5 \sqrt{23})^{1 / 2}\left(\frac{25+3 \sqrt{69}}{2}\right)^{3 / 4} \\
& =\left(\frac{5+\sqrt{23}}{\sqrt{2}}\right)(36 \sqrt{3}+13 \sqrt{23})^{1 / 2}=\left(\frac{5+\sqrt{23}}{\sqrt{2}}\right)\left(\frac{3 \sqrt{3}+\sqrt{23}}{2}\right)^{3 / 2} .
\end{aligned}
$$

By Lemma 3.3,

$$
P^{-1}=\frac{1}{4 \sqrt{2}}\left(Q+Q^{-1}\right)+\frac{1}{4 \sqrt{2}} \sqrt{\left(Q+Q^{-1}\right)^{2}+32} .
$$

From (4.8),

$$
Q+Q^{-1}=\sqrt{Q^{2}+Q^{-2}+2}=\sqrt{16(187+108 \sqrt{3})},
$$


and, from (4.10),

$$
\left(Q+Q^{-1}\right)^{2}+32=16(9+6 \sqrt{3})^{2} .
$$

Putting these calculations in (4.9), we find that

$$
P^{-1}=\frac{1}{\sqrt{2}} \sqrt{187+108 \sqrt{3}}+\frac{1}{\sqrt{2}}(9+6 \sqrt{3}) .
$$

By (4.8),

$$
G_{69}=Q^{1 / 12} P^{-1 / 6}=\left(\frac{5+\sqrt{23}}{\sqrt{2}}\right)^{1 / 12}\left(\frac{3 \sqrt{3}+\sqrt{23}}{2}\right)^{1 / 8} P^{-1 / 6},
$$

and thus, by (4.11), it remains to show that

$$
\left(\sqrt{\frac{6+3 \sqrt{3}}{4}}+\sqrt{\frac{2+3 \sqrt{3}}{4}}\right)^{3}=\frac{1}{\sqrt{2}} \sqrt{187+108 \sqrt{3}}+\frac{1}{\sqrt{2}}(9+6 \sqrt{3}) .
$$

This can be achieved by a straightforward computation.

Theorem 4.3.

\begin{tabular}{|c|c|c|c|c|c|c|c|c|c|c|}
\hline$d_{1}$ & $d_{2}$ & $\chi$ & $\boldsymbol{G}$ & $C$ & $\begin{array}{l}\chi\left(\boldsymbol{G}_{0}\right) \\
\chi\left(\boldsymbol{G}_{2}\right)\end{array}$ & $\begin{array}{l}\chi\left(\boldsymbol{G}_{1}\right) \\
\chi\left(\boldsymbol{G}_{3}\right)\end{array}$ & $h_{1}$ & $h_{2}$ & $w_{2}$ & $\epsilon_{1}$ \\
\hline 1 & -308 & $\chi_{0}$ & $\boldsymbol{G}_{0}$ & $\begin{array}{c}{[1, \Omega]} \\
{[14,7+\Omega]}\end{array}$ & $\begin{array}{l}1 \\
1\end{array}$ & $\begin{array}{l}1 \\
1\end{array}$ & & & & \\
\hline 28 & -11 & $\chi_{1}$ & $\boldsymbol{G}_{1}$ & $\begin{array}{c}{[2,1+\Omega]} \\
{[7, \Omega]}\end{array}$ & $\begin{array}{l}1 \\
1\end{array}$ & $\begin{array}{l}-1 \\
-1\end{array}$ & 1 & 1 & 2 & $8+3 \sqrt{7}$ \\
\hline 77 & -4 & $\chi_{2}$ & $\boldsymbol{G}_{2}$ & $\begin{array}{c}{[3,1+\Omega]} \\
{[3,-1+\Omega]}\end{array}$ & $\begin{array}{r}1 \\
-1\end{array}$ & $\begin{array}{r}-1 \\
1\end{array}$ & 1 & 1 & 4 & $\frac{9+\sqrt{77}}{2}$ \\
\hline 44 & -7 & $\chi_{3}$ & $\boldsymbol{G}_{3}$ & $\begin{array}{c}{[6,1+\Omega]} \\
{[6,-1+\Omega]}\end{array}$ & $\begin{array}{r}1 \\
-1 \\
\end{array}$ & $\begin{array}{r}1 \\
-1\end{array}$ & & & & \\
\hline
\end{tabular}

$$
G_{77}=(8+3 \sqrt{7})^{1 / 8}\left(\frac{\sqrt{11}+\sqrt{7}}{2}\right)^{1 / 8}\left(\sqrt{\frac{6+\sqrt{11}}{4}}+\sqrt{\frac{2+\sqrt{11}}{4}}\right)^{1 / 2} .
$$

Proof. We compose the following table giving needed information about ideal classes and characters.

We see from the table that 77 is of the first kind. Thus, by Theorem 3.1, since $h=8$ and $w=2$,

$$
Q:=\left(\frac{G_{77}}{G_{11 / 7}}\right)^{4}=(8+3 \sqrt{7})\left(\frac{9+\sqrt{77}}{2}\right)^{1 / 2}=(8+3 \sqrt{7})\left(\frac{\sqrt{11}+\sqrt{7}}{2}\right)
$$

If $P=\left(G_{77} G_{11 / 7}\right)^{-3}$, then, from Lemma 3.3,

$$
P^{-1}=\frac{Q+Q^{-1}+7+\sqrt{\left(Q+Q^{-1}+7\right)^{2}-32}}{4 \sqrt{2}}
$$

Now

$$
Q+Q^{-1}+7=8 \sqrt{11}+28=2 \sqrt{2}(2 \sqrt{22}+7 \sqrt{2})
$$


Using this in (4.13), we find that

$$
P^{-1}=\frac{1}{2}(2 \sqrt{22}+7 \sqrt{2}+\sqrt{182+56 \sqrt{11}}) .
$$

By (4.12),

$$
G_{77}=Q^{1 / 8} P^{-1 / 6}=(8+3 \sqrt{7})^{1 / 8}\left(\frac{\sqrt{11}+\sqrt{7}}{2}\right)^{1 / 8} P^{-1 / 6},
$$

and thus by (4.14) it remains to show that

$$
\left(\sqrt{\frac{6+\sqrt{11}}{4}}+\sqrt{\frac{2+\sqrt{11}}{4}}\right)^{3}=\frac{1}{2}(2 \sqrt{22}+7 \sqrt{2}+\sqrt{182+56 \sqrt{11}}),
$$

which is readily shown by a straightforward calculation.

\section{Theorem 4.4.}

$$
G_{141}=(4 \sqrt{3}+\sqrt{47})^{1 / 8}\left(\frac{7+\sqrt{47}}{\sqrt{2}}\right)^{1 / 12}\left(\sqrt{\frac{18+9 \sqrt{3}}{4}}+\sqrt{\frac{14+9 \sqrt{3}}{4}}\right)^{1 / 2} .
$$

Proof. We record the necessary information in the following table.

\begin{tabular}{|c|c|c|c|c|rc|c|c|c|c|}
\hline$d_{1}$ & $d_{2}$ & $\chi$ & $\boldsymbol{G}$ & $C$ & $\begin{array}{r}\chi\left(\boldsymbol{G}_{0}\right) \\
\chi\left(\boldsymbol{G}_{2}\right)\end{array}$ & $\begin{array}{r}\chi\left(\boldsymbol{G}_{1}\right) \\
\chi\left(\boldsymbol{G}_{3}\right)\end{array}$ & $h_{1}$ & $h_{2}$ & $w_{2}$ & $\epsilon_{1}$ \\
\hline 1 & -564 & $\chi_{0}$ & $\boldsymbol{G}_{0}$ & $\begin{array}{c}{[1, \Omega]} \\
{[6,3+\Omega]}\end{array}$ & $\begin{array}{r}1 \\
1\end{array}$ & $\begin{array}{r}1 \\
1\end{array}$ & & & & \\
\hline 188 & -3 & $\chi_{1}$ & $\boldsymbol{G}_{1}$ & $\begin{array}{c}{[2,1+\Omega]} \\
{[3, \Omega]}\end{array}$ & $\begin{array}{r}1 \\
-1\end{array}$ & $\begin{array}{r}-1 \\
1\end{array}$ & 1 & 1 & 6 & $48+7 \sqrt{47}$ \\
\hline 141 & -4 & $\chi_{2}$ & $\boldsymbol{G}_{2}$ & $\begin{array}{c}{[5,2+\Omega]} \\
{[5,-2+\Omega]}\end{array}$ & $\begin{array}{r}1 \\
1\end{array}$ & -1 & 1 & 1 & 4 & $95+8 \sqrt{141}$ \\
\hline 12 & -47 & $\chi_{3}$ & $\boldsymbol{G}_{3}$ & $\begin{array}{c}1 \\
{[10,3+\Omega]} \\
{[10,-3+\Omega]}\end{array}$ & $\begin{array}{r}1 \\
-1\end{array}$ & -1 & & & & \\
\hline
\end{tabular}

We see that 141 is again of the first kind. Applying Theorem 3.1, we find that, since $h=8$ and $w=2$,

$$
\left(\frac{G_{141}}{G_{47 / 3}}\right)^{4}=(48+7 \sqrt{47})^{1 / 3}(95+8 \sqrt{141})^{1 / 2} .
$$

Let $Q=\left(G_{141} / G_{47 / 3}\right)^{6}$. Then, by (4.15),

$$
\begin{aligned}
Q=(48+7 \sqrt{47})^{1 / 2}(95+8 \sqrt{141})^{3 / 4} & =\left(\frac{7+\sqrt{47}}{\sqrt{2}}\right)(4 \sqrt{3}+\sqrt{47})^{3 / 2} \\
& =(48+7 \sqrt{47})^{1 / 2}(756 \sqrt{3}+191 \sqrt{47})^{1 / 2} .
\end{aligned}
$$

Let $P=\left(G_{141} G_{47 / 3}\right)^{-3}$. Then, by Lemma 3.3,

$$
P^{-1}=\frac{\left(Q+Q^{-1}\right)+\sqrt{\left(Q+Q^{-1}\right)^{2}+32}}{4 \sqrt{2}} .
$$


From the last representation of $Q$ in (4.16),

$$
Q^{2}+Q^{-2}+34=(36(7+4 \sqrt{3}))^{2},
$$

and so

$$
Q+Q^{-1}=\sqrt{Q^{2}+Q^{-2}+2}=4 \sqrt{7855+4536 \sqrt{3}} .
$$

Using these calculations in (4.17), we deduce that

$$
P^{-1}=\frac{1}{\sqrt{2}} \sqrt{7855+4536 \sqrt{3}}+\frac{9}{\sqrt{2}}(7+4 \sqrt{3}) .
$$

Hence, by (4.16) and (4.18),

$$
\begin{aligned}
G_{141}=Q^{1 / 12} P^{-1 / 6}= & \left(\frac{7+\sqrt{47}}{\sqrt{2}}\right)^{1 / 12}(4 \sqrt{3}+\sqrt{47})^{1 / 8} \\
& \times\left(\frac{1}{\sqrt{2}} \sqrt{7855+4536 \sqrt{3}}+\frac{9}{\sqrt{2}}(7+4 \sqrt{3})\right)^{1 / 6} .
\end{aligned}
$$

It thus remains to show that

$$
\left(\sqrt{\frac{18+9 \sqrt{3}}{4}}+\sqrt{\frac{14+9 \sqrt{3}}{4}}\right)^{3}=\frac{1}{\sqrt{2}} \sqrt{7855+4536 \sqrt{3}}+\frac{9}{\sqrt{2}}(7+4 \sqrt{3})
$$

\begin{tabular}{|c|c|c|c|c|c|c|c|c|c|c|}
\hline$d_{1}$ & $d_{2}$ & $\chi$ & $\boldsymbol{G}$ & C & $\begin{array}{l}\chi\left(\boldsymbol{G}_{0}\right) \\
\chi\left(\boldsymbol{G}_{2}\right) \\
\end{array}$ & $\begin{array}{l}\chi\left(\boldsymbol{G}_{1}\right) \\
\chi\left(\boldsymbol{G}_{3}\right)\end{array}$ & $h_{1}$ & $h_{2}$ & $w_{2}$ & $\epsilon_{1}$ \\
\hline 1 & -580 & $\chi_{0}$ & $\boldsymbol{G}_{0}$ & $\begin{array}{l}{[1, \Omega]} \\
{[5, \Omega]}\end{array}$ & $\begin{array}{l}1 \\
1 \\
\end{array}$ & $\begin{array}{l}1 \\
1\end{array}$ & & & & \\
\hline 29 & -20 & $\chi_{1}$ & $\boldsymbol{G}_{1}$ & $\begin{array}{c}{[2,1+\Omega]} \\
{[10,5+\Omega]}\end{array}$ & $\begin{array}{l}1 \\
1 \\
\end{array}$ & $\begin{array}{l}-1 \\
-1 \\
\end{array}$ & 1 & 2 & 2 & $\frac{\sqrt{29}+5}{2}$ \\
\hline 5 & -116 & $\chi_{2}$ & $\boldsymbol{G}_{2}$ & $\begin{array}{c}{[7,3+\Omega]} \\
{[7,-3+\Omega]}\end{array}$ & $\begin{array}{r}1 \\
-1\end{array}$ & $\begin{array}{r}-1 \\
1\end{array}$ & 1 & 6 & 2 & $\frac{\sqrt{5}+1}{2}$ \\
\hline 145 & -4 & $\chi_{3}$ & $\boldsymbol{G}_{3}$ & $\begin{array}{c}{[11,3+\Omega]} \\
{[11,-3+\Omega]}\end{array}$ & $\begin{array}{r}1 \\
-1\end{array}$ & $\begin{array}{r}1 \\
-1\end{array}$ & & & & \\
\hline
\end{tabular}

which is a straightforward, albeit laborious, task.

Theorem 4.5.

$$
G_{145}=(\sqrt{5}+2)^{1 / 4}\left(\frac{\sqrt{29}+5}{2}\right)^{1 / 4}\left(\sqrt{\frac{17+\sqrt{145}}{8}}+\sqrt{\frac{9+\sqrt{145}}{8}}\right)^{1 / 2} .
$$

Proof. We compose the following table.

Thus, 145 is of the second kind. Thus, by Theorem 3.2, since $h=8$ and $w=2$,

$$
\left(G_{145} G_{29 / 5}\right)^{4}=\left(\frac{\sqrt{29}+5}{2}\right)^{2}\left(\frac{\sqrt{5}+1}{2}\right)^{6}=\left(\frac{\sqrt{29}+5}{2}\right)^{2}(\sqrt{5}+2)^{2} .
$$

Hence,

$$
P^{-1}:=\left(G_{145} G_{29 / 5}\right)^{2}=\left(\frac{\sqrt{29}+5}{2}\right)(\sqrt{5}+2) .
$$


By Lemma 3.4, with $Q=\left(G_{145} / G_{29 / 5}\right)^{3}$, we have

$$
Q=P^{-1}-P+\sqrt{\left(P^{-1}-P\right)^{2}-1} .
$$

By (4.19), we readliy find that

$$
P^{-1}-P=2 \sqrt{29}+5 \sqrt{5},
$$

and so, by (4.20),

$$
Q=2 \sqrt{29}+5 \sqrt{5}+\sqrt{240+20 \sqrt{145}} .
$$

Thus, by (4.19) and (4.21),

$$
\begin{aligned}
G_{145}=P^{-1 / 4} Q^{1 / 6}= & \left(\frac{\sqrt{29}+5}{2}\right)^{1 / 4}(\sqrt{5}+2)^{1 / 4} \\
& \times(2 \sqrt{29}+5 \sqrt{5}+\sqrt{240+20 \sqrt{145}})^{1 / 6} .
\end{aligned}
$$

Hence, it remains to show that

$$
2 \sqrt{29}+5 \sqrt{5}+\sqrt{240+20 \sqrt{145}}=\left(\sqrt{\frac{17+\sqrt{145}}{8}}+\sqrt{\frac{9+\sqrt{145}}{8}}\right)^{3},
$$

which is readily shown.

Theorem 4.6.

$$
G_{205}=\left(\frac{\sqrt{5}+1}{2}\right)\left(\frac{3 \sqrt{5}+\sqrt{41}}{2}\right)^{1 / 4}\left(\sqrt{\frac{7+\sqrt{41}}{8}}+\sqrt{\frac{\sqrt{41}-1}{8}}\right) .
$$

Proof. We record the following table.

\begin{tabular}{|c|c|c|c|c|rr|c|c|c|c|}
\hline$d_{1}$ & $d_{2}$ & $\chi$ & $\boldsymbol{G}$ & $C$ & $\begin{array}{r}\chi\left(\boldsymbol{G}_{0}\right) \\
\chi\left(\boldsymbol{G}_{2}\right)\end{array}$ & $\chi\left(\boldsymbol{G}_{1}\right)$ & $h_{1}$ & $h_{2}$ & $w_{2}$ & $\epsilon_{1}$ \\
\hline 1 & -820 & $\chi_{0}$ & $\boldsymbol{G}_{0}$ & $\begin{array}{c}{[1, \Omega]} \\
{[5, \Omega]}\end{array}$ & $\begin{array}{r}1 \\
1\end{array}$ & 1 & & & & \\
\hline 5 & -164 & $\chi_{1}$ & $\boldsymbol{G}_{1}$ & $\begin{array}{c}{[2,1+\Omega]} \\
{[10,5+\Omega]}\end{array}$ & $\begin{array}{r}1 \\
1\end{array}$ & -1 & 1 & 8 & 2 & $\frac{\sqrt{5}+1}{2}$ \\
\hline 205 & -4 & $\chi_{2}$ & $\boldsymbol{G}_{2}$ & $\begin{array}{c}{[11,2+\Omega]} \\
{[11,-2+\Omega]}\end{array}$ & $\begin{array}{r}1 \\
-1\end{array}$ & -1 & 2 & 1 & 4 & $\frac{43+3 \sqrt{205}}{2}$ \\
\hline 41 & -20 & $\chi_{3}$ & $\boldsymbol{G}_{3}$ & $\begin{array}{c}{[13,4+\Omega]} \\
{[13,-4+\Omega]}\end{array}$ & $\begin{array}{r}1 \\
-1\end{array}$ & -1 & & & & \\
\hline
\end{tabular}

Note that 205 is of the second kind. Applying Theorem 3.2 with $h=8$ and $w=2$, we deduce that

$$
\begin{aligned}
P^{-2}:=\left(G_{205} G_{41 / 5}\right)^{4} & =\left(\frac{\sqrt{5}+1}{2}\right)^{8}\left(\frac{43+3 \sqrt{205}}{2}\right) \\
& =\left(\frac{7+3 \sqrt{5}}{2}\right)^{2}\left(\frac{3 \sqrt{5}+\sqrt{41}}{2}\right)^{2} .
\end{aligned}
$$


Letting $Q=\left(G_{205} / G_{41 / 5}\right)^{3}$, we deduce from Lemma 3.4 that

$$
Q=\left(P^{-1}-P\right)+\sqrt{\left(P^{-1}-P\right)^{2}-1} .
$$

From (4.22),

$$
P^{-1}-P=\frac{45+7 \sqrt{41}}{2}
$$

Thus, from (4.23),

$$
Q=\frac{1}{2}(45+7 \sqrt{41}+\sqrt{4030+630 \sqrt{41}}) .
$$

If follows from (4.22) and (4.24) that

$$
\begin{aligned}
G_{205}=P^{-1 / 4} Q^{1 / 6}= & \left(\frac{\sqrt{5}+1}{2}\right)\left(\frac{3 \sqrt{5}+\sqrt{41}}{2}\right)^{1 / 4} \\
& \times\left(\frac{1}{2}(45+7 \sqrt{41}+\sqrt{4030+630 \sqrt{41}})\right)^{1 / 6} .
\end{aligned}
$$

It thus remains to show that

$$
\frac{1}{2}(45+7 \sqrt{41}+\sqrt{4030+630 \sqrt{41}})=\left(\sqrt{\frac{7+\sqrt{41}}{8}}+\sqrt{\frac{\sqrt{41}-1}{8}}\right)^{6} .
$$

\begin{tabular}{|c|c|c|c|c|c|c|c|c|c|c|}
\hline$d_{1}$ & $d_{2}$ & $\chi$ & $\boldsymbol{G}$ & $C$ & $\begin{array}{l}\chi\left(\boldsymbol{G}_{0}\right) \\
\chi\left(\boldsymbol{G}_{2}\right) \\
\end{array}$ & $\begin{array}{l}\chi\left(\boldsymbol{G}_{1}\right) \\
\chi\left(\boldsymbol{G}_{3}\right)\end{array}$ & $h_{1}$ & $h_{2}$ & $w_{2}$ & $\epsilon_{1}$ \\
\hline 1 & -852 & $\chi_{0}$ & $\boldsymbol{G}_{0}$ & $\begin{array}{c}{[1, \Omega]} \\
{[6,3+\Omega]} \\
\end{array}$ & $\begin{array}{l}1 \\
1 \\
\end{array}$ & $\begin{array}{l}1 \\
1 \\
\end{array}$ & & & & \\
\hline 284 & -3 & $\chi_{1}$ & $\boldsymbol{G}_{1}$ & $\begin{array}{c}{[2,1+\Omega]} \\
{[3, \Omega]}\end{array}$ & $\begin{array}{l}1 \\
1\end{array}$ & $\begin{array}{l}-1 \\
-1\end{array}$ & 1 & 1 & 6 & $3480+413 \sqrt{71}$ \\
\hline 213 & -4 & $\chi_{2}$ & $\boldsymbol{G}_{2}$ & $\begin{array}{c}{[7,2+\Omega]} \\
{[7,-2+\Omega]}\end{array}$ & $\begin{array}{r}1 \\
-1\end{array}$ & $\begin{array}{r}-1 \\
1\end{array}$ & 1 & 1 & 4 & $\frac{73+5 \sqrt{213}}{2}$ \\
\hline 12 & -71 & $\chi_{3}$ & $\boldsymbol{G}_{3}$ & $\begin{array}{c}{[14,5+\Omega]} \\
{[14,-5+\Omega]}\end{array}$ & $\begin{array}{r}1 \\
-1\end{array}$ & $\begin{array}{r}1 \\
-1\end{array}$ & & & & \\
\hline
\end{tabular}

This is more readily accomplished if we first note that

$$
\left(\sqrt{\frac{7+\sqrt{41}}{8}}+\sqrt{\frac{\sqrt{41}-1}{8}}\right)^{2}=\frac{\sqrt{41}+3}{4}+\sqrt{\frac{17+3 \sqrt{41}}{8}} .
$$

\section{Theorem 4.7.}

$G_{213}=\left(\frac{5 \sqrt{3}+\sqrt{71}}{2}\right)^{1 / 8}\left(\frac{59+7 \sqrt{71}}{\sqrt{2}}\right)^{1 / 12}\left(\sqrt{\frac{21+12 \sqrt{3}}{2}}+\sqrt{\frac{19+12 \sqrt{3}}{2}}\right)^{1 / 2}$.

Proof. We have the following table. 
Observe that 213 is of the first kind. Applying Theorem 3.1 with $h=8$ and $w=2$, we find that

$$
\begin{aligned}
Q^{2 / 3}:=\left(\frac{G_{213}}{G_{71 / 3}}\right)^{4} & =(3480+413 \sqrt{71})^{1 / 3}\left(\frac{73+5 \sqrt{213}}{2}\right)^{1 / 2} \\
& =\left(\frac{59+7 \sqrt{71}}{\sqrt{2}}\right)^{2 / 3}\left(\frac{5 \sqrt{3}+\sqrt{71}}{2}\right)
\end{aligned}
$$

so that

$$
Q=\left(\frac{59+7 \sqrt{71}}{\sqrt{2}}\right)\left(\frac{5 \sqrt{3}+\sqrt{71}}{2}\right)^{3 / 2}=\left(\frac{59+7 \sqrt{71}}{\sqrt{2}}\right)(180 \sqrt{3}+37 \sqrt{21})^{1 / 2} .
$$

Let $P=\left(G_{213} G_{71 / 3}\right)^{-3}$. Then, by Lemma 3.3,

$$
P^{-1}=\frac{1}{4 \sqrt{2}}\left(\left(Q+Q^{-1}\right)+\sqrt{\left(Q+Q^{-1}\right)^{2}+32}\right) .
$$

By (4.25) and moderate calculations,

$$
\left(Q+Q^{-1}\right)^{2}+32=(12(87+50 \sqrt{3}))^{2}
$$

and

$$
\frac{1}{4 \sqrt{2}}\left(Q+Q^{-1}\right)=\frac{1}{4 \sqrt{2}} \sqrt{Q^{2}+Q^{-2}+2}=\sqrt{\frac{1}{2}(135619+78300 \sqrt{3})} .
$$

Thus, by (4.26),

$$
P^{-1}=\sqrt{\frac{1}{2}(135619+78300 \sqrt{3})}+\frac{3}{\sqrt{2}}(87+50 \sqrt{3}) .
$$

Thus, by (4.25) and (4.27),

$$
\begin{aligned}
G_{213}=Q^{1 / 12} P^{-1 / 6}= & \left(\frac{5 \sqrt{3}+\sqrt{71}}{2}\right)^{1 / 8}\left(\frac{59+7 \sqrt{71}}{\sqrt{2}}\right)^{1 / 12} \\
& \times\left(\sqrt{\frac{1}{2}(135619+78300 \sqrt{3})}+\frac{3}{\sqrt{2}}(87+50 \sqrt{3})\right)^{1 / 6} .
\end{aligned}
$$

Hence, it remains to show that

$\sqrt{\frac{1}{2}(135619+78300 \sqrt{3})}+\frac{3}{\sqrt{2}}(87+50 \sqrt{3})=\left(\sqrt{\frac{21+12 \sqrt{3}}{2}}+\sqrt{\frac{19+12 \sqrt{3}}{2}}\right)^{3}$,

which is accomplished by a direct calculation.

Theorem 4.8.

$$
G_{265}=(\sqrt{5}+2)^{1 / 4}\left(\frac{\sqrt{53}+7}{2}\right)^{1 / 4}\left(\sqrt{\frac{89+5 \sqrt{265}}{8}}+\sqrt{\frac{81+5 \sqrt{265}}{8}}\right)^{1 / 2} .
$$

Proof. The following table is easily verified. 


\begin{tabular}{|c|c|c|c|c|rr|c|c|c|c|}
\hline$d_{1}$ & $d_{2}$ & $\chi$ & $\boldsymbol{G}$ & $C$ & $\begin{array}{r}\chi\left(\boldsymbol{G}_{0}\right) \\
\chi\left(\boldsymbol{G}_{2}\right)\end{array}$ & $\begin{array}{r}\chi\left(\boldsymbol{G}_{1}\right) \\
\chi\left(\boldsymbol{G}_{3}\right)\end{array}$ & $h_{1}$ & $h_{2}$ & $w_{2}$ & $\epsilon_{1}$ \\
\hline 1 & -1060 & $\chi_{0}$ & $\boldsymbol{G}_{0}$ & $\begin{array}{c}{[1, \Omega]} \\
{[10,5+\Omega]}\end{array}$ & $\begin{array}{r}1 \\
1\end{array}$ & $\begin{array}{l}1 \\
1\end{array}$ & & & & \\
\hline 5 & -212 & $\chi_{1}$ & $\boldsymbol{G}_{1}$ & $\begin{array}{c}{[2,1+\Omega]} \\
{[5, \Omega]}\end{array}$ & $\begin{array}{r}-1 \\
-1\end{array}$ & 1 & 1 & 6 & 2 & $\frac{\sqrt{5}+1}{2}$ \\
\hline 53 & -20 & $\chi_{2}$ & $\boldsymbol{G}_{2}$ & $\begin{array}{c}{[7,1+\Omega]} \\
{[7,-1+\Omega]}\end{array}$ & $\begin{array}{r}1 \\
1\end{array}$ & -1 & 1 & 2 & 2 & $\frac{\sqrt{53}+7}{2}$ \\
\hline 265 & -4 & $\chi_{3}$ & $\boldsymbol{G}_{3}$ & $\begin{array}{c}{[14,1+\Omega]} \\
{[14,-1+\Omega]}\end{array}$ & $\begin{array}{r}1 \\
-1\end{array}$ & -1 & & & & \\
\hline
\end{tabular}

Note that 265 is of the first kind. Applying Theorem 3.1 with $h=8$ and $w=2$, we find that

$$
Q^{4 / 3}:=\left(\frac{G_{265}}{G_{53 / 5}}\right)^{4}=\left(\frac{\sqrt{5}+1}{2}\right)^{6}\left(\frac{\sqrt{53}+7}{2}\right)^{2}
$$

so that

$$
Q=\left(\frac{\sqrt{5}+1}{2}\right)^{9 / 2}\left(\frac{\sqrt{53}+7}{2}\right)^{3 / 2}=(38+17 \sqrt{5})^{1 / 2}(182+25 \sqrt{53})^{1 / 2} .
$$

Let $P=\left(G_{265} G_{53 / 5}\right)^{-2}$. Then, by Lemma 3.4 ,

$$
P^{-1}=\frac{\left(Q+Q^{-1}\right)+\sqrt{\left(Q+Q^{-1}\right)^{2}+16}}{4} .
$$

By using (4.28) and the identity $Q+Q^{-1}=\sqrt{Q^{2}+Q^{-2}+2}$ in (4.29), we find that

$$
P^{-1}=\frac{1}{2 \sqrt{2}} \sqrt{6917+425 \sqrt{265}}+\frac{1}{4}(85+5 \sqrt{265}) .
$$

By (4.28) and (4.30),

$$
\begin{aligned}
G_{265}=Q^{1 / 6} P^{-1 / 4}= & (\sqrt{5}+2)^{1 / 4}\left(\frac{\sqrt{53}+7}{2}\right)^{1 / 4} \\
& \times\left(\frac{1}{2 \sqrt{2}} \sqrt{6917+425 \sqrt{265}}+\frac{1}{4}(85+5 \sqrt{265})\right)^{1 / 4} .
\end{aligned}
$$

Hence, it remains to show that

$$
\frac{1}{2 \sqrt{2}} \sqrt{6917+425 \sqrt{265}}+\frac{1}{4}(85+5 \sqrt{265})=\left(\sqrt{\frac{89+5 \sqrt{265}}{8}}+\sqrt{\frac{81+5 \sqrt{265}}{8}}\right)^{2}
$$

which is easy to establish.

\section{Theorem 4.9.}

$$
G_{301}=(8+3 \sqrt{7})^{1 / 8}\left(\frac{23 \sqrt{43}+57 \sqrt{7}}{2}\right)^{1 / 8}\left(\sqrt{\frac{46+7 \sqrt{43}}{4}}+\sqrt{\frac{42+7 \sqrt{43}}{4}}\right)^{1 / 2} .
$$

Proof. We compose the following table. 


\begin{tabular}{|c|c|c|c|c|rr|c|c|c|c|}
\hline$d_{1}$ & $d_{2}$ & $\chi$ & $\boldsymbol{G}$ & $C$ & $\begin{array}{r}\chi\left(\boldsymbol{G}_{0}\right) \\
\chi\left(\boldsymbol{G}_{2}\right)\end{array}$ & $\begin{array}{c}\chi\left(\boldsymbol{G}_{1}\right) \\
\chi\left(\boldsymbol{G}_{3}\right)\end{array}$ & $h_{1}$ & $h_{2}$ & $w_{2}$ & $\epsilon_{1}$ \\
\hline 1 & -1204 & $\chi_{0}$ & $\boldsymbol{G}_{0}$ & $\begin{array}{c}{[1, \Omega]} \\
{[14,7+\Omega]}\end{array}$ & $\begin{array}{r}1 \\
1\end{array}$ & $\begin{array}{r}1 \\
1\end{array}$ & & & & \\
\hline 28 & -43 & $\chi_{1}$ & $\boldsymbol{G}_{1}$ & $\begin{array}{c}{[2,1+\Omega]} \\
{[7, \Omega]}\end{array}$ & $\begin{array}{r}1 \\
-1\end{array}$ & -1 & 1 & 1 & 2 & $8+3 \sqrt{7}$ \\
\hline 301 & -4 & $\chi_{2}$ & $\boldsymbol{G}_{2}$ & $\begin{array}{c}{[5,2+\Omega]} \\
{[5,-2+\Omega]}\end{array}$ & $\begin{array}{r}1 \\
1\end{array}$ & -1 & 1 & 1 & 4 & $\frac{22745+1311 \sqrt{301}}{1}$ \\
\hline 172 & -7 & $\chi_{3}$ & $\boldsymbol{G}_{3}$ & $\begin{array}{c}{[10,3+\Omega]} \\
{[10,-3+\Omega]}\end{array}$ & $\begin{array}{r}1 \\
-1\end{array}$ & -1 & & & \\
\hline
\end{tabular}

Thus, 301 is of the first kind. Applying Theorem 3.1 with $h=8$ and $w=2$, we find that

$$
\begin{aligned}
Q:=\left(\frac{G_{301}}{G_{43 / 7}}\right)^{4} & =(8+3 \sqrt{7})\left(\frac{22745+1311 \sqrt{301}}{2}\right)^{1 / 2} \\
& =(8+3 \sqrt{7})\left(\frac{23 \sqrt{43}+57 \sqrt{7}}{2}\right) .
\end{aligned}
$$

Let $P=\left(G_{301} G_{43 / 7}\right)^{-3}$. Then, by Lemma 3.5 and (4.31),

$$
\begin{aligned}
P^{-1} & =\frac{1}{4 \sqrt{2}}\left(Q+Q^{-1}+7\right)+\frac{1}{4 \sqrt{2}} \sqrt{\left(Q+Q^{-1}+7\right)^{2}-32} \\
& =\frac{1}{\sqrt{2}}(301+46 \sqrt{43})+\frac{1}{\sqrt{2}} \sqrt{7(25941+3956 \sqrt{43})} .
\end{aligned}
$$

Therefore, by (4.31) and (4.32),

$$
\begin{aligned}
G_{301}=Q^{1 / 8} P^{-1 / 6}= & (8+3 \sqrt{7})^{1 / 8}\left(\frac{23 \sqrt{43}+57 \sqrt{7}}{2}\right)^{1 / 8} \\
& \times\left(\frac{1}{\sqrt{2}}(301+46 \sqrt{43})+\frac{1}{\sqrt{2}} \sqrt{7(25941+3956 \sqrt{43})}\right)^{1 / 6} .
\end{aligned}
$$

It remains to show that

$$
\begin{gathered}
\frac{1}{\sqrt{2}}(301+46 \sqrt{43})+\frac{1}{\sqrt{2}} \sqrt{7(25941+3956 \sqrt{43})} \\
=\left(\sqrt{\frac{46+7 \sqrt{43}}{4}}+\sqrt{\frac{42+7 \sqrt{43}}{4}}\right)^{3}
\end{gathered}
$$

which is a routine task.

\section{Theorem 4.10.}

$$
G_{445}=(\sqrt{5}+2)^{1 / 2}\left(\frac{\sqrt{445}+21}{2}\right)^{1 / 4}\left(\sqrt{\frac{13+\sqrt{89}}{8}}+\sqrt{\frac{5+\sqrt{89}}{8}}\right) .
$$

Proof. We form the following table. 


\begin{tabular}{|c|c|c|c|c|c|c|c|c|c|c|}
\hline$d_{1}$ & $d_{2}$ & $\chi$ & $\boldsymbol{G}$ & $C$ & $\begin{array}{l}\chi\left(\boldsymbol{G}_{0}\right) \\
\chi\left(\boldsymbol{G}_{2}\right) \\
\end{array}$ & $\begin{array}{l}\chi\left(\boldsymbol{G}_{1}\right) \\
\chi\left(\boldsymbol{G}_{3}\right) \\
\end{array}$ & $h_{1}$ & $h_{2}$ & $w_{2}$ & $\epsilon_{1}$ \\
\hline 1 & -1780 & $\chi_{0}$ & $\boldsymbol{G}_{0}$ & $\begin{array}{l}{[1, \Omega]} \\
{[5, \Omega]} \\
\end{array}$ & $\begin{array}{l}1 \\
1\end{array}$ & $\begin{array}{l}1 \\
1\end{array}$ & & & & \\
\hline 5 & -356 & $\chi_{1}$ & $\boldsymbol{G}_{1}$ & $\begin{array}{c}{[2,1+\Omega]} \\
{[10,5+\Omega]} \\
\end{array}$ & $\begin{array}{r}1 \\
-1 \\
\end{array}$ & $\begin{array}{r}-1 \\
1 \\
\end{array}$ & 1 & 12 & 2 & $\frac{\sqrt{5}+1}{2}$ \\
\hline 445 & -4 & $\chi_{2}$ & $\boldsymbol{G}_{2}$ & $\begin{array}{c}{[13,6+\Omega]} \\
{[13,-6+\Omega]}\end{array}$ & $\begin{array}{l}1 \\
1\end{array}$ & $\begin{array}{l}-1 \\
-1 \\
\end{array}$ & 4 & 1 & 4 & $\frac{\sqrt{445}+21}{2}$ \\
\hline 89 & -20 & $\chi_{3}$ & $\boldsymbol{G}_{3}$ & $\begin{array}{c}{[19,7+\Omega]} \\
{[19,-7+\Omega]}\end{array}$ & $\begin{array}{r}1 \\
-1\end{array}$ & $\begin{array}{r}1 \\
-1\end{array}$ & & & & \\
\hline
\end{tabular}

Thus, 445 is of the second kind. Applying Theorem 3.2 with $h=8$ and $w=2$, we deduce that

$$
P^{-2}:=\left(G_{445} G_{89 / 5}\right)^{4}=\left(\frac{\sqrt{5}+1}{2}\right)^{12}\left(\frac{\sqrt{445}+21}{2}\right)^{2},
$$

so that

$$
P^{-1}=(9+4 \sqrt{5})\left(\frac{\sqrt{445}+21}{2}\right) .
$$

Let $Q=\left(G_{445} / G_{89 / 5}\right)^{3}$. Then, by Lemma 3.4 and (4.33),

$$
Q=\left(P^{-1}-P\right)+\sqrt{\left(P^{-1}-P\right)^{2}-1}=189+20 \sqrt{89}+\sqrt{71320+7560 \sqrt{89}} .
$$

Therefore, by (4.33) and (4.34),

$$
\begin{aligned}
G_{445}=P^{-1 / 4} Q^{1 / 6}= & (9+4 \sqrt{5})^{1 / 4}\left(\frac{\sqrt{445}+21}{2}\right)^{1 / 4} \\
& \times(189+20 \sqrt{89}+\sqrt{71320+7560 \sqrt{89}})^{1 / 6} .
\end{aligned}
$$

It thus remains to show that

$$
189+20 \sqrt{89}+\sqrt{71320+7560 \sqrt{89}}=\left(\sqrt{\frac{13+\sqrt{89}}{8}}+\sqrt{\frac{5+\sqrt{89}}{8}}\right)^{6}
$$

By first squaring the binomial on the right side and then cubing the resulting expression, we can easily verify the desired equality.

\section{Theorem 4.11.}

$$
\begin{aligned}
G_{505} & =(\sqrt{5}+2)^{1 / 2}\left(\frac{\sqrt{5}+1}{2}\right)^{1 / 4}(\sqrt{101}+10)^{1 / 4} \\
& \times\left(\sqrt{\frac{113+5 \sqrt{505}}{8}}+\sqrt{\frac{105+5 \sqrt{505}}{8}}\right)^{1 / 2} .
\end{aligned}
$$

Proof. We compose the following table. 


\begin{tabular}{|c|c|c|c|c|c|c|c|c|c|c|}
\hline$d_{1}$ & $d_{2}$ & $\chi$ & $G$ & $C$ & $\begin{array}{l}\chi\left(\boldsymbol{G}_{0}\right) \\
\chi\left(\boldsymbol{G}_{2}\right)\end{array}$ & $\begin{array}{l}\chi\left(\boldsymbol{G}_{1}\right) \\
\chi\left(\boldsymbol{G}_{3}\right)\end{array}$ & $h_{1}$ & $h_{2}$ & $w_{2}$ & $\epsilon_{1}$ \\
\hline 1 & -2020 & $\chi_{0}$ & $\boldsymbol{G}_{0}$ & $\begin{array}{l}{[1, \Omega]} \\
{[5, \Omega]}\end{array}$ & $\begin{array}{l}1 \\
1\end{array}$ & $\begin{array}{l}1 \\
1\end{array}$ & & & & \\
\hline 5 & -404 & $\chi_{1}$ & $\boldsymbol{G}_{1}$ & $\begin{array}{c}{[2,1+\Omega]} \\
{[10,5+\Omega]}\end{array}$ & $\begin{array}{l}1 \\
1\end{array}$ & $\begin{array}{l}-1 \\
-1\end{array}$ & 1 & 14 & 2 & $\frac{\sqrt{5}+1}{2}$ \\
\hline 101 & -20 & $\chi_{2}$ & $\boldsymbol{G}_{2}$ & $\begin{array}{c}{[11,1+\Omega]} \\
{[11,-1+\Omega]}\end{array}$ & $\begin{array}{r}1 \\
-1\end{array}$ & $\begin{array}{r}-1 \\
1\end{array}$ & 1 & 2 & 2 & $\sqrt{101}+10$ \\
\hline 505 & -4 & $\chi_{3}$ & $\boldsymbol{G}_{3}$ & $\begin{array}{c}{[22,1+\Omega]} \\
{[22,-1+\Omega]}\end{array}$ & $\begin{array}{r}1 \\
-1\end{array}$ & $\begin{array}{r}1 \\
-1\end{array}$ & & & & \\
\hline
\end{tabular}

Hence, 505 is of the second kind. Applying Theorem 3.2 with $h=8$ and $w=2$, we find that

$$
P^{-2}:=\left(G_{505} G_{101 / 5}\right)^{4}=\left(\frac{\sqrt{5}+1}{2}\right)^{14}(\sqrt{101}+10)^{2}
$$

so that

$$
\begin{aligned}
P^{-1}=\left(\frac{\sqrt{5}+1}{2}\right)^{7}(\sqrt{101}+10) & =(\sqrt{5}+2)\left(\frac{\sqrt{5}+1}{2}\right)^{4}(\sqrt{101}+10) \\
& =(\sqrt{5}+2)\left(\frac{7+3 \sqrt{5}}{2}\right)(\sqrt{101}+10)
\end{aligned}
$$

Let $Q=\left(G_{505} / G_{101 / 5}\right)^{3}$. Then, by Lemma 3.4 and (4.35),

$$
\begin{aligned}
Q & =\left(P^{-1}-P\right)+\sqrt{\left(P^{-1}-P\right)^{2}-1} \\
& =(130 \sqrt{5}+29 \sqrt{101})+\sqrt{169440+7540 \sqrt{505}} .
\end{aligned}
$$

Therefore, by (4.35) and (4.36),

$$
\begin{aligned}
G_{505}=P^{-1 / 4} Q^{1 / 6}= & (\sqrt{5}+2)^{1 / 2}\left(\frac{\sqrt{5}+1}{2}\right)^{1 / 4}(\sqrt{101}+10)^{1 / 4} \\
& \times((130 \sqrt{5}+29 \sqrt{101})+\sqrt{169440+7540 \sqrt{505}})^{1 / 6} .
\end{aligned}
$$

Thus, it remains to show that

$$
(130 \sqrt{5}+29 \sqrt{101})+\sqrt{169440+7540 \sqrt{505}}=\left(\sqrt{\frac{113+5 \sqrt{505}}{8}}+\sqrt{\frac{105+5 \sqrt{505}}{8}}\right)^{3},
$$

which is straightforward.

\section{5. $G_{217}$ AND $G_{553}$}

The genus structures for $\mathbb{Q}(\sqrt{-217})$ and $\mathbb{Q}(\sqrt{-553})$ are different from those for the eleven imaginary quadratic fields addressed in Section 4 , and so $G_{217}$ and $G_{553}$ must be calculated by another means. This we accomplish in this section. 
Lemma 5.1. Let $m$ denote a positive integer with $7 \mid m$. Let $\tau=\sqrt{-m} / 7$ and $Q=$ $\left(G_{m} / G_{m / 49}\right)^{4}$. Then

$$
\left(\frac{\eta(\tau) \eta\left(\frac{\tau+1}{2}\right)}{\eta(7 \tau) \eta\left(\frac{7 \tau+1}{2}\right)}\right)^{2}-49\left(\frac{\eta(\tau) \eta\left(\frac{\tau+1}{2}\right)}{\eta(7 \tau) \eta\left(\frac{7 \tau+1}{2}\right)}\right)^{-2}=Q^{3 / 2}+8 Q^{1 / 2}-8 Q^{-1 / 2}-Q^{-3 / 2} .
$$

Proof. With $q=\exp (-\pi \sqrt{m} / 7)$, it follows from (2.3) that

$$
\left(\frac{\eta(\tau) \eta\left(\frac{\tau+1}{2}\right)}{\eta(7 \tau) \eta\left(\frac{7 \tau+1}{2}\right)}\right)^{2}=\frac{f^{2}\left(-q^{2}\right) f^{2}(q)}{q^{3 / 2} f^{2}\left(-q^{14}\right) f^{2}\left(q^{7}\right)} .
$$

Next, by an entry from Ramanujan's second notebook [2, p. 209, Entry 55],

$$
\begin{aligned}
& \frac{f^{2}(-q) f^{2}\left(-q^{2}\right)}{q^{3 / 2} f^{2}\left(-q^{7}\right) f^{2}\left(-q^{14}\right)}+49 \frac{q^{3 / 2} f^{2}\left(-q^{7}\right) f^{2}\left(-q^{14}\right)}{f^{2}(-q) f^{2}\left(-q^{2}\right)}=\frac{f^{6}\left(-q^{2}\right) f^{6}\left(-q^{7}\right)}{q^{3 / 2} f^{6}(-q) f^{6}\left(-q^{14}\right)} \\
& -8 \frac{f^{2}\left(-q^{2}\right) f^{2}\left(-q^{7}\right)}{q^{1 / 2} f^{2}(-q) f^{2}\left(-q^{14}\right)}-8 \frac{q^{1 / 2} f^{2}(-q) f^{2}\left(-q^{14}\right)}{f^{2}\left(-q^{2}\right) f^{2}\left(-q^{7}\right)}+\frac{q^{3 / 2} f^{6}(-q) f^{6}\left(-q^{14}\right)}{f^{6}\left(-q^{2}\right) f^{6}\left(-q^{7}\right)}
\end{aligned}
$$

Multiplying both sides by $q^{3 / 2}$ and then replacing $q$ by $-q$, we find that

$$
\begin{aligned}
& \frac{f^{2}(q) f^{2}\left(-q^{2}\right)}{f^{2}\left(q^{7}\right) f^{2}\left(-q^{14}\right)}-49 \frac{q^{3} f^{2}\left(q^{7}\right) f^{2}\left(-q^{14}\right)}{f^{2}(q) f^{2}\left(-q^{2}\right)}=\frac{f^{6}\left(-q^{2}\right) f^{6}\left(q^{7}\right)}{f^{6}(q) f^{6}\left(-q^{14}\right)} \\
& +8 \frac{q f^{2}\left(-q^{2}\right) f^{2}\left(q^{7}\right)}{f^{2}(q) f^{2}\left(-q^{14}\right)}-8 \frac{q^{2} f^{2}(q) f^{2}\left(-q^{14}\right)}{f^{2}\left(-q^{2}\right) f^{2}\left(q^{7}\right)}-\frac{q^{3} f^{6}(q) f^{6}\left(-q^{14}\right)}{f^{6}\left(-q^{2}\right) f^{6}\left(q^{7}\right)} .
\end{aligned}
$$

Recall that $q=\exp (-\pi \sqrt{m} / 7)$ and recall that $G_{m / 49}$ is then given by (1.2). Thus, $G_{m}=2^{-1 / 4} q^{-7 / 24}\left(-q^{7} ; q^{14}\right)_{\infty}$. Hence,

$$
\left(\frac{G_{m}}{G_{m / 49}}\right)^{2}=\frac{\left(-q^{7} ; q^{14}\right)_{\infty}^{2}}{q^{1 / 2}\left(-q ; q^{2}\right)_{\infty}^{2}}=\frac{\left(q^{2} ; q^{2}\right)_{\infty}^{2}\left(-q^{7} ;-q^{7}\right)_{\infty}^{2}}{q^{1 / 2}(-q ;-q)_{\infty}^{2}\left(q^{14} ; q^{14}\right)_{\infty}^{2}}=\frac{f^{2}\left(-q^{2}\right) f^{2}\left(q^{7}\right)}{q^{1 / 2} f^{2}(q) f^{2}\left(-q^{14}\right)}
$$

Dividing (5.3) by $q^{3 / 2}$ and substituting (5.2) and (5.4) into the resulting equality, we deduce (5.1) to complete the proof.

Theorem 5.2. Let $m$ be a squarefree positive integer with $7 \mid m$ and $m \equiv 1(\bmod 4)$. Let $K=\mathbb{Q}(\sqrt{-m})$ be an imaginary quadratic field such that each genus contains exactly two classes and such that the principal genus $\boldsymbol{G}_{0}$ comprises $[1, \Omega]$ and $[2,1+$ $\Omega]$, while $[7, \Omega]$ and $[14,7+\Omega]$ form a nonprincipal genus $\boldsymbol{G}_{1}$. Then

$$
\left\{\frac{1}{\sqrt{7}}\left(\frac{\eta(\tau) \eta\left(\frac{\tau+1}{2}\right)}{\eta(7 \tau) \eta\left(\frac{7 \tau+1}{2}\right)}\right)\right\}^{h / 2}=\prod_{\chi\left(\boldsymbol{G}_{1}\right)=-1} \epsilon_{1}^{w h_{1} h_{2} / w_{2}},
$$

where $h, h_{1}$, and $h_{2}$ are the class numbers of $K, \mathbb{Q}\left(\sqrt{d_{1}}\right)$, and $\mathbb{Q}\left(\sqrt{d_{2}}\right)$, respectively, $w$ and $w_{2}$ are the numbers of roots of unity in $K$ and $\mathbb{Q}\left(\sqrt{d_{2}}\right)$, respectively, $\epsilon_{1}$ is the fundamental unit in $\mathbb{Q}\left(\sqrt{d_{1}}\right)$, and the product is over all characters $\chi$ (with $\chi\left(\boldsymbol{G}_{1}\right)=$ -1 ), associated with the decomposition $d=d_{1} d_{2}$, and therefore $d_{1}, d_{2}, h_{1}, h_{2}, w_{2}$, and $\epsilon_{1}$ are dependent on $\chi$. 
Proof. Let $A_{0}=[1, \Omega], A_{0}^{\prime}=[2,1+\Omega], A_{1}=[7, \Omega]$, and $A_{1}^{\prime}=[14,7+\Omega]$. Then by the same reasoning that we used in the proof of Theorem 3.1,

$$
\left(\frac{F\left(A_{1}\right) F\left(A_{1}^{\prime}\right)}{F\left(A_{0}\right) F\left(A_{0}^{\prime}\right)}\right)^{h / 4}=\prod_{\chi\left(\boldsymbol{G}_{1}\right)=-1} \epsilon_{1}^{w h_{1} h_{2} / w_{2}} .
$$

By (2.17),

$$
\begin{aligned}
& F\left(A_{0}\right)=\eta^{2}(\Omega)=\eta^{2}(7 \tau), \\
& F\left(A_{0}^{\prime}\right)=\eta^{2}\left(\frac{\Omega+1}{2}\right) / \sqrt{2}=\eta^{2}\left(\frac{7 \tau+1}{2}\right) / \sqrt{2}, \\
& F\left(A_{1}\right)=\eta^{2}\left(\frac{\Omega}{7}\right) / \sqrt{7}=\eta^{2}(\tau) / \sqrt{7},
\end{aligned}
$$

and

$$
F\left(A_{1}^{\prime}\right)=\eta^{2}\left(\frac{\Omega+7}{14}\right) / \sqrt{14}=\eta^{2}\left(\frac{\tau+1}{2}\right) / \sqrt{14} .
$$

Substituting these values into (5.6) and recalling that the number of genus charac-

\begin{tabular}{|c|c|c|c|c|c|c|c|c|c|c|}
\hline$d_{1}$ & $d_{2}$ & $\chi$ & $G$ & $C$ & $\begin{array}{l}\chi\left(\boldsymbol{G}_{0}\right) \\
\chi\left(\boldsymbol{G}_{2}\right)\end{array}$ & $\begin{array}{l}\chi\left(\boldsymbol{G}_{1}\right) \\
\chi\left(\boldsymbol{G}_{3}\right)\end{array}$ & $h_{1}$ & $h_{2}$ & $w_{2}$ & $\epsilon_{1}$ \\
\hline 1 & -868 & $\chi_{0}$ & $\boldsymbol{G}_{0}$ & $\begin{array}{c}{[1, \Omega]} \\
{[2,1+\Omega]}\end{array}$ & $\begin{array}{l}1 \\
1\end{array}$ & $\begin{array}{l}1 \\
1\end{array}$ & & & & \\
\hline 124 & -7 & $\chi_{1}$ & $\boldsymbol{G}_{1}$ & $\begin{array}{c}{[7, \Omega]} \\
{[14,7+\Omega]}\end{array}$ & $\begin{array}{r}1 \\
-1 \\
\end{array}$ & $\begin{array}{r}-1 \\
1 \\
\end{array}$ & 1 & 1 & 2 & $1520+273 \sqrt{31}$ \\
\hline 217 & -4 & $\chi_{2}$ & $G_{2}$ & $\begin{array}{c}{[11,5+\Omega]} \\
{[11,-5+\Omega]}\end{array}$ & $\begin{array}{r}1 \\
-1 \\
\end{array}$ & $\begin{array}{r}1 \\
-1 \\
\end{array}$ & 1 & 1 & 4 & $3844063+260952 \sqrt{217}$ \\
\hline 28 & -31 & $\chi_{3}$ & $G_{3}$ & $\begin{array}{c}{[13,2+\Omega]} \\
{[13,-2+\Omega]}\end{array}$ & $\begin{array}{l}1 \\
1\end{array}$ & $\begin{array}{l}-1 \\
-1\end{array}$ & & & & \\
\hline
\end{tabular}
ters $\chi$ with $\chi\left(\boldsymbol{G}_{1}\right)=-1$ is equal to $h / 4$, we deduce (5.5) to complete the proof.

Theorem 5.3.

$$
G_{217}=\left(\sqrt{\frac{11+4 \sqrt{7}}{2}}+\sqrt{\frac{9+4 \sqrt{7}}{2}}\right)^{1 / 2}\left(\sqrt{\frac{16+5 \sqrt{7}}{4}}+\sqrt{\frac{12+5 \sqrt{7}}{4}}\right)^{1 / 2} .
$$

Proof. We set up a table to summarize some information that we need.

It is clear that $\mathbb{Q}(\sqrt{-217})$ satisfies the conditions of Theorem 5.2. Thus, since $h=8$ and $w=2$, we deduce that

$$
\begin{aligned}
\frac{1}{7^{2}}\left(\frac{\eta(\tau) \eta\left(\frac{\tau+1}{2}\right)}{\eta(7 \tau) \eta\left(\frac{7 \tau+1}{2}\right)}\right)^{4} & =(1520+273 \sqrt{31})(3844063+260952 \sqrt{217})^{1 / 2} \\
& =(1520+273 \sqrt{31})(524 \sqrt{7}+249 \sqrt{31}),
\end{aligned}
$$

so that

$$
\left(\frac{\eta(\tau) \eta\left(\frac{\tau+1}{2}\right)}{\eta(7 \tau) \eta\left(\frac{7 \tau+1}{2}\right)}\right)^{2}=7 \epsilon
$$

where

$$
\epsilon=(1520+273 \sqrt{31})^{1 / 2}(524 \sqrt{7}+249 \sqrt{31})^{1 / 2} .
$$


It follows from (5.1) that

$$
Q^{3 / 2}+8 Q^{1 / 2}-8 Q^{-1 / 2}-Q^{3 / 2}=7\left(\epsilon-\epsilon^{-1}\right),
$$

where $Q=\left(G_{217} / G_{31 / 7}\right)^{4}$. By an elementary calculation,

$$
\begin{aligned}
\left(\epsilon-\epsilon^{-1}\right)^{2} & =\epsilon^{2}+\epsilon^{-2}-2 \\
& =4(1053643+398240 \sqrt{7}) \\
& =4(27+10 \sqrt{7})^{2}(367+140 \sqrt{7}) .
\end{aligned}
$$

Let $x=Q^{1 / 2}-Q^{-1 / 2}$. Then (5.8) can be recast in the form

$$
\begin{aligned}
x^{3}+11 x & =14(27+10 \sqrt{7}) \sqrt{367+140 \sqrt{7}} \\
& =(367+140 \sqrt{7}) \sqrt{367+140 \sqrt{7}}+11 \sqrt{367+140 \sqrt{7}} .
\end{aligned}
$$

It is now obvious that

$$
x=\sqrt{367+140 \sqrt{7}} .
$$

Solving (5.9) for $Q^{1 / 2}$, we find that

$$
\begin{aligned}
Q^{1 / 2} & =\frac{1}{2}(\sqrt{367+140 \sqrt{7}}+\sqrt{371+140 \sqrt{7}}) \\
& =\frac{1}{2}(\sqrt{367+140 \sqrt{7}}+(14+5 \sqrt{7})) \\
& =\left(\sqrt{\frac{16+5 \sqrt{7}}{4}}+\sqrt{\frac{12+5 \sqrt{7}}{4}}\right)^{2} .
\end{aligned}
$$

Now let $P=\left(G_{217} G_{31 / 7}\right)^{-3}$. Using Lemma 3.5 and (5.9), we deduce that

$$
\begin{aligned}
P+P^{-1} & =\frac{1}{2 \sqrt{2}}\left(Q+Q^{-1}+7\right) \\
& =\frac{1}{2 \sqrt{2}}\left(x^{2}+9\right)=\frac{1}{2 \sqrt{2}}(376+140 \sqrt{7}) .
\end{aligned}
$$

Solving for $P^{-1}$, we find that

$$
\begin{aligned}
P^{-1} & =\frac{94+35 \sqrt{7}}{\sqrt{2}}+\sqrt{\frac{17409+6580 \sqrt{7}}{2}} \\
& =\left(\sqrt{\frac{11+4 \sqrt{7}}{2}}+\sqrt{\frac{9+4 \sqrt{7}}{2}}\right)^{3} .
\end{aligned}
$$

Thus, from (5.10) and (5.11),

$$
\begin{aligned}
G_{217}=P^{-1 / 6} Q^{1 / 8}= & \left(\sqrt{\frac{11+4 \sqrt{7}}{2}}+\sqrt{\frac{9+4 \sqrt{7}}{2}}\right)^{1 / 2} \\
& \times\left(\sqrt{\frac{16+5 \sqrt{7}}{4}}+\sqrt{\frac{12+5 \sqrt{7}}{4}}\right)^{1 / 2},
\end{aligned}
$$

which completes the proof. 
Theorem 5.4.

$$
\begin{aligned}
G_{553}= & \left(\sqrt{\frac{100+11 \sqrt{79}}{4}}+\sqrt{\frac{96+11 \sqrt{79}}{4}}\right)^{1 / 2} \\
& \times\left(\sqrt{\frac{143+16 \sqrt{79}}{2}}+\sqrt{\frac{141+16 \sqrt{79}}{2}}\right)^{1 / 2} .
\end{aligned}
$$

Proof. We set up the following table to summarize the information that we need.

\begin{tabular}{|c|c|c|c|c|rr|c|c|c|c|}
\hline$d_{1}$ & $d_{2}$ & $\chi$ & $\boldsymbol{G}$ & $C$ & $\begin{array}{r}\chi\left(\boldsymbol{G}_{0}\right) \\
\chi\left(\boldsymbol{G}_{2}\right)\end{array}$ & $\begin{array}{r}\chi\left(\boldsymbol{G}_{1}\right) \\
\chi\left(\boldsymbol{G}_{3}\right)\end{array}$ & $h_{1}$ & $h_{2}$ & $w_{2}$ & $\epsilon_{1}$ \\
\hline 1 & -2212 & $\chi_{0}$ & $\boldsymbol{G}_{0}$ & $\begin{array}{c}{[1, \Omega]} \\
{[2,1+\Omega]}\end{array}$ & $\begin{array}{r}1 \\
1\end{array}$ & 1 & & & & \\
\hline 28 & -79 & $\chi_{1}$ & $\boldsymbol{G}_{1}$ & $\begin{array}{c}{[7, \Omega]} \\
{[14,7+\Omega]}\end{array}$ & $\begin{array}{r}1 \\
-1\end{array}$ & $\begin{array}{r}-1 \\
1\end{array}$ & 1 & 5 & 2 & $8+3 \sqrt{7}$ \\
\hline 553 & -4 & $\chi_{2}$ & $\boldsymbol{G}_{2}$ & $\begin{array}{c}{[17,5+\Omega]} \\
{[17,-5+\Omega]}\end{array}$ & $\begin{array}{r}1 \\
1\end{array}$ & -1 & 1 & 1 & 4 & $\begin{array}{r}6 \\
+1\end{array}$ \\
\hline 316 & -7 & $\chi_{3}$ & $\boldsymbol{G}_{3}$ & $\begin{array}{c}{[19,6+\Omega]} \\
{[19,-6+\Omega]}\end{array}$ & $\begin{array}{r}1 \\
-1\end{array}$ & -1 & & & & \\
\hline
\end{tabular}

It is clear that $\mathbb{Q}(\sqrt{-553})$ satisfies the hypotheses of Theorem 5.2. Thus, since $h=8$ and $w=2$,

$\frac{1}{7^{2}}\left(\frac{\eta(\tau) \eta\left(\frac{\tau+1}{2}\right)}{\eta(7 \tau) \eta\left(\frac{7 \tau+1}{2}\right)}\right)^{4}=(8+3 \sqrt{7})^{5}(624,635,837,407+26,562,217,704 \sqrt{553})^{1 / 2}$

so that

$$
\left(\frac{\eta(\tau) \eta\left(\frac{\tau+1}{2}\right)}{\eta(7 \tau) \eta\left(\frac{7 \tau+1}{2}\right)}\right)^{2}=7 \epsilon
$$

where

$$
\epsilon=(514,088+194,307 \sqrt{7})^{1 / 2}(211,227 \sqrt{7}+62,876 \sqrt{79})^{1 / 2} .
$$

Then an elementary calculation gives

$$
\begin{aligned}
\left(\epsilon-\epsilon^{-1}\right)^{2} & =\epsilon^{2}+\epsilon^{-2}-2 \\
& =4(143,650,096,411+16,161,898,544 \sqrt{79}) \\
& =(391+44 \sqrt{79})(19170+2156 \sqrt{79})^{2} .
\end{aligned}
$$

By Lemma 5.1 and (5.12)-(5.14), with $Q=\left(G_{553} / G_{79 / 7}\right)^{4}$,

$$
\begin{aligned}
Q^{3 / 2}+8 Q^{1 / 2}-8 Q^{-1 / 2}-Q^{-3 / 2} & =7\left(\epsilon-\epsilon^{-1}\right) \\
& =7 \sqrt{391+44 \sqrt{79}}(19170+2156 \sqrt{79}) .
\end{aligned}
$$

If $x=Q^{1 / 2}-Q^{-1 / 2}$, then the foregoing equality may be written in the form

$$
x^{3}+11 x=7 \sqrt{391+44 \sqrt{79}}\left(7^{2}(391+44 \sqrt{79})+11\right),
$$


from which it is obvious that

$$
x=Q^{1 / 2}-Q^{-1 / 2}=7 \sqrt{391+44 \sqrt{79}} .
$$

Solving for $Q^{1 / 2}$, we readily find that

$$
\begin{aligned}
Q^{1 / 2} & =\frac{1}{2}(7 \sqrt{391+44 \sqrt{79}}+(98+11 \sqrt{79})) \\
& =\left(\sqrt{\frac{100+11 \sqrt{79}}{4}}+\sqrt{\frac{96+11 \sqrt{79}}{4}}\right)^{2} .
\end{aligned}
$$

Now let $P=\left(G_{553} G_{79 / 7}\right)^{-3}$. Then, by Lemma 3.5 and (5.15),

$$
2 \sqrt{2}\left(P+P^{-1}\right)=Q+Q^{-1}+7=x^{2}+9=19168+2156 \sqrt{79} .
$$

Solving for $P^{-1}$, we find that

$$
\begin{aligned}
P^{-1} & =\frac{1}{\sqrt{2}}(4792+539 \sqrt{79}+\sqrt{45,914,421+5,165,776 \sqrt{79}}) \\
& =\left(\sqrt{\frac{143+16 \sqrt{79}}{2}}+\sqrt{\frac{141+16 \sqrt{79}}{2}}\right)^{3} .
\end{aligned}
$$

Thus, by (5.16) and (5.17),

$$
\begin{aligned}
G_{553}=Q^{1 / 8} P^{-1 / 6}= & \left(\sqrt{\frac{100+11 \sqrt{79}}{4}}+\sqrt{\frac{96+11 \sqrt{79}}{4}}\right)^{1 / 2} \\
& \times\left(\sqrt{\frac{143+16 \sqrt{79}}{2}}+\sqrt{\frac{141+16 \sqrt{79}}{2}}\right)^{1 / 2},
\end{aligned}
$$

and the proof is complete.

\section{Class Invariants and Modular Equations}

In this section we establish six of Ramanujan's class invariants by using tools well known to Ramanujan, in particular, modular equations.

Second Proof of Theorem 4.1. From (1.1) and (2.3) it is easily seen that

$$
\frac{f(-q)}{f\left(-q^{2}\right)}=\chi(-q) \text {. }
$$

Using this equality, we rewrite two of Ramanujan's eta-function identities in terms of $\chi$. Thus [2, pp. 206, 211]

$$
\begin{aligned}
& \frac{f(-q) f\left(-q^{2}\right)}{q^{3 / 2} f\left(-q^{13}\right) f\left(-q^{26}\right)}+13 \frac{q^{3 / 2} f\left(-q^{13}\right) f\left(-q^{26}\right)}{f(-q) f\left(-q^{2}\right)}=\left(q^{-1 / 2} \frac{\chi\left(-q^{13}\right)}{\chi(-q)}\right)^{3} \\
& -4\left(q^{-1 / 2} \frac{\chi\left(-q^{13}\right)}{\chi(-q)}\right)-4\left(q^{1 / 2} \frac{\chi(-q)}{\chi\left(-q^{13}\right)}\right)+\left(q^{1 / 2} \frac{\chi(-q)}{\chi\left(-q^{13}\right)}\right)^{3}
\end{aligned}
$$


and

(6.3)

$\frac{f(-q) f\left(-q^{2}\right)}{q^{1 / 2} f\left(-q^{5}\right) f\left(-q^{10}\right)}+5 \frac{q^{1 / 2} f\left(-q^{5}\right) f\left(-q^{10}\right)}{f(-q) f\left(-q^{2}\right)}=\left(q^{-1 / 6} \frac{\chi\left(-q^{5}\right)}{\chi(-q)}\right)^{3}+\left(q^{1 / 6} \frac{\chi(-q)}{\chi\left(-q^{5}\right)}\right)^{3}$.

Replace $q$ by $-q$ in (6.2) and then set $q=\exp (-\pi \sqrt{5 / 13})$. If

$$
A:=e^{(3 \pi / 2) \sqrt{5 / 13}} \frac{f\left(e^{-\pi \sqrt{5 / 13}}\right) f\left(-e^{-2 \pi \sqrt{5 / 13}}\right)}{f\left(e^{-\pi \sqrt{65}}\right) f\left(-e^{-2 \pi \sqrt{65}}\right)}
$$

and

$$
B:=e^{(\pi / 2) \sqrt{5 / 13}} \frac{\chi\left(e^{-\pi \sqrt{65}}\right)}{\chi\left(e^{-\pi \sqrt{5 / 13}}\right)},
$$

then $(6.2)$ can be recast in the form

$$
A-13 A^{-1}=B^{3}+4 B-4 B^{-1}-B^{-3} .
$$

Next, replace $q$ by $-q$ in $(6.3)$ and then set $q=\exp (-\pi \sqrt{13 / 5})$. If

$$
A^{\prime}:=e^{(\pi / 2) \sqrt{13 / 5}} \frac{f\left(e^{-\pi \sqrt{13 / 5}}\right) f\left(-e^{-2 \pi \sqrt{13 / 5}}\right)}{f\left(e^{-\pi \sqrt{65}}\right) f\left(-e^{-2 \pi \sqrt{65}}\right)}
$$

and

$$
B^{\prime}:=e^{(\pi / 6) \sqrt{13 / 5}} \frac{\chi\left(e^{-\pi \sqrt{65}}\right)}{\chi\left(e^{-\pi \sqrt{13 / 5}}\right)},
$$

then (6.3) takes the shape

$$
A^{\prime}-5 A^{\prime-1}=B^{\prime 3}-B^{\prime-3} \text {. }
$$

We shall prove that

$$
B=B^{\prime} \quad \text { and } \quad A=\sqrt{\frac{13}{5}} A^{\prime} .
$$

Now, by (1.2),

$$
G_{n}=2^{-1 / 4} e^{\pi \sqrt{n} / 24} \chi\left(e^{-\pi \sqrt{n}}\right) \quad \text { and } \quad G_{1 / n}=2^{-1 / 4} e^{\pi /(24 \sqrt{n})} \chi\left(e^{-\pi / \sqrt{n}}\right) .
$$

Since $G_{n}=G_{1 / n}$, we find that

$$
\chi\left(e^{-\pi \sqrt{n}}\right)=e^{(\pi / 24)(1 / \sqrt{n}-\sqrt{n})} \chi\left(e^{-\pi / \sqrt{n}}\right) .
$$

(This could also be proved by using (6.1) along with the transformation formula for $f$.) In particular, if $n=5 / 13,(6.11)$ yields the equality

$$
\chi\left(e^{-\pi \sqrt{5 / 13}}\right)=e^{\pi /(3 \sqrt{65})} \chi\left(e^{-\pi \sqrt{13 / 5}}\right) .
$$

The aforementioned transformation formula for $f(-q)$ is given by [1, p. 43, Entry $27($ iii)]

$$
e^{-a / 12} a^{1 / 4} f\left(-e^{-2 a}\right)=e^{-b / 12} b^{1 / 4} f\left(-e^{-2 b}\right),
$$

where $a, b>0$ with $a b=\pi^{2}$. If $a=\pi \sqrt{5 / 13}$, so that $b=\pi \sqrt{13 / 5}$, then we deduce from (6.13) that

$$
f\left(-e^{-2 \pi \sqrt{5 / 13}}\right)=(13 / 5)^{1 / 4} e^{-2 \pi /(3 \sqrt{65})} f\left(-e^{-2 \pi \sqrt{13 / 5}}\right) .
$$


First, from (6.5) and (6.12),

$$
B=\frac{e^{(\pi / 2) \sqrt{5 / 13}} \chi\left(e^{-\pi \sqrt{65}}\right)}{e^{\pi /(3 \sqrt{65})} \chi\left(e^{-\pi \sqrt{13 / 5}}\right)}=e^{(\pi / 6) \sqrt{13 / 5}} \frac{\chi\left(e^{-\pi \sqrt{65}}\right)}{\chi\left(e^{-\pi \sqrt{13 / 5}}\right)}=B^{\prime},
$$

by (6.8). Thus, the first equality of (6.10) has been demonstrated. Second, by (6.4), (6.1) with $q=-\exp (-\pi \sqrt{5 / 13}),(6.12),(6.14)$, and lastly (6.1) with $q=$ $\exp (-\pi \sqrt{13 / 5})$

$$
\begin{aligned}
& A=e^{(3 \pi / 2) \sqrt{5 / 13}} \frac{\chi\left(e^{-\pi \sqrt{5 / 13}}\right) f^{2}\left(-e^{-2 \pi \sqrt{5 / 13}}\right)}{f\left(e^{-\pi \sqrt{65}}\right) f\left(-e^{-2 \pi \sqrt{65}}\right)} \\
& =\sqrt{\frac{13}{5}} e^{(\pi / 2) \sqrt{13 / 5}} \frac{\chi\left(e^{-\pi \sqrt{13 / 5}}\right) f^{2}\left(-e^{-2 \pi \sqrt{13 / 5}}\right)}{f\left(e^{-\pi \sqrt{65}}\right) f\left(-e^{-2 \pi \sqrt{65}}\right)} \\
& =\sqrt{\frac{13}{5}} e^{(\pi / 2) \sqrt{13 / 5}} \frac{f\left(e^{-\pi \sqrt{13 / 5}}\right) f\left(-e^{-2 \pi \sqrt{13 / 5}}\right)}{f\left(e^{-\pi \sqrt{65}}\right) f\left(-e^{-2 \pi \sqrt{65}}\right)} \\
& =\sqrt{\frac{13}{5}} A^{\prime},
\end{aligned}
$$

by (6.7). Thus, the second equality of (6.10) has been established.

Employing (6.10) in (6.9), we find that

$$
\sqrt{\frac{5}{13}} A-\sqrt{65} A^{-1}=B^{3}-B^{-3}=\left(B-B^{-1}\right)^{3}+3\left(B-B^{-1}\right) .
$$

Dividing both sides by $u:=B-B^{-1}(\neq 0)$, we find that

$$
\frac{\sqrt{65}}{13}\left(u^{2}+7\right)=u^{2}+3 \text {. }
$$

Solving for $u^{2}$, we find that $u^{2}=(\sqrt{65}-1) / 2$. Thus, since clearly $B>1$,

$$
B-B^{-1}=\sqrt{\frac{\sqrt{65}-1}{2}} .
$$

Now solving for $B$, we find that

$$
B=\sqrt{\frac{\sqrt{65}-1}{8}}+\sqrt{\frac{\sqrt{65}+7}{8}}
$$

where in solving the quadratic equation we took the plus sign since $B>0$.

If $q=\exp (-\pi \sqrt{13 / 5})$, then $q^{5}=\exp (-\pi \sqrt{65})$. Hence, from (1.2) and (6.5), we readily see that $B=G_{65} / G_{13 / 5}$. Furthermore, from (1.6), $G_{13 / 5}=\{4 \alpha(1-\alpha)\}^{-1 / 24}$. Hence, if $\beta$ has degree 5 over $\alpha$, then $G_{65}=\{4 \beta(1-\beta)\}^{-1 / 24}$.

We now employ Lemma 3.4, where it is to be noted that $P=\left(G_{65} G_{13 / 5}\right)^{-2}$ and $Q=B^{-3}=\left(G_{65} / G_{13 / 5}\right)^{-3}$. We already know $Q$ from (6.15). To determine $P$ from Lemma 3.4, we first calculate

$$
\begin{aligned}
Q+Q^{-1} & =B^{3}+B^{-3}=\left(B+B^{-1}\right)\left\{\left(B+B^{-1}\right)^{2}-3\right\} \\
& =\sqrt{\frac{\sqrt{65}+7}{2}}\left(\frac{\sqrt{65}+7}{2}-3\right)=\sqrt{74+10 \sqrt{65}}
\end{aligned}
$$


Thus, using (6.16) in Lemma 3.4 and solving for $P^{-1}$, we find that

$$
P^{-1}=\frac{1}{4}(\sqrt{74+10 \sqrt{65}}+\sqrt{90+10 \sqrt{65}}),
$$

since $P>0$.

Hence, by (6.15) and (6.17),

$$
\begin{aligned}
G_{65}=B^{1 / 2} P^{-1 / 4}= & \left(\sqrt{\frac{\sqrt{65}+7}{8}}+\sqrt{\frac{\sqrt{65}-1}{8}}\right)^{1 / 2} \\
& \times\left(\frac{1}{4}(\sqrt{74+10 \sqrt{65}}+\sqrt{90+10 \sqrt{65}})\right)^{1 / 4} .
\end{aligned}
$$

We must show that (6.18) can be transformed into the form of Theorem 4.1. First,

$$
\begin{aligned}
\frac{1}{2}(\sqrt{74+10 \sqrt{65}}+\sqrt{90+10 \sqrt{65}})^{1 / 2} & =\frac{1}{2}(\sqrt{(9+\sqrt{65})(1+\sqrt{65})}+5+\sqrt{65})^{1 / 2} \\
& =\sqrt{\frac{9+\sqrt{65}}{8}}+\sqrt{\frac{1+\sqrt{65}}{8}} .
\end{aligned}
$$

Second,

$$
\begin{aligned}
\left(\sqrt{\frac{\sqrt{65}+7}{8}}+\sqrt{\left.\frac{\sqrt{65}-1}{8}\right)^{2}}\right. & =\frac{1}{4}(3+\sqrt{65}+\sqrt{58+6 \sqrt{65}}) \\
& =\frac{1}{4}(3+\sqrt{65}+3 \sqrt{5}+\sqrt{13}) \\
& =\left(\frac{\sqrt{13}+3}{2}\right)\left(\frac{\sqrt{5}+1}{2}\right) .
\end{aligned}
$$

Putting (6.19) and (6.20) in (6.18), we complete the proof.

Before commencing our second proof of Theorem 4.2, we establish a general principle. Let $p$ and $r$ denote coprime, positive integers. Set $q=\exp (-\pi \sqrt{p / r})$ and $q^{\prime}=\exp (-\pi \sqrt{p r})$, and let $\beta$ have degree $r$ over $\alpha$. Then, by (1.6),

$$
G_{p / r}=\{4 \alpha(1-\alpha)\}^{-1 / 24} \quad \text { and } \quad G_{p r}=\{4 \beta(1-\beta)\}^{-1 / 24} .
$$

Furthermore, from (1.4) and (1.5),

$$
\frac{K(\sqrt{1-\alpha})}{K(\sqrt{\alpha})}=\sqrt{\frac{p}{r}}
$$

and from the defintion (1.3) of a modular equation,

$$
r \frac{K(\sqrt{1-\alpha})}{K(\sqrt{\alpha})}=\frac{K(\sqrt{1-\beta})}{K(\sqrt{\beta})} .
$$

If we solve (6.22) for $r$ and substitute this in (6.23), we find that

$$
p \frac{K(\sqrt{\alpha})}{K(\sqrt{1-\alpha})}=\frac{K(\sqrt{1-\beta})}{K(\sqrt{\beta})} .
$$


From the last equality we conclude:

If $\beta$ has degree $r$ over $\alpha$, then $\beta$ has degree $p$ over $1-\alpha$.

Furthermore, from (1.7) and (6.22),

$$
\frac{\varphi^{2}\left(e^{-\pi \sqrt{p / r}}\right)}{\varphi^{2}\left(e^{-\pi \sqrt{r / p}}\right)}=\frac{K(\sqrt{\alpha})}{K(\sqrt{1-\alpha})}=\sqrt{\frac{r}{p}} .
$$

Second Proof of Theorem 4.2. We need two of Ramanujan's modular equations of degree 23 [1, p. 411, Entry 15(i), (ii)]. If $\beta$ has degree 23 over $\alpha$, then

$$
(\alpha \beta)^{1 / 8}+\{(1-\alpha)(1-\beta)\}^{1 / 8}+2^{2 / 3}\{\alpha \beta(1-\alpha)(1-\beta)\}^{1 / 24}=1
$$

and

$$
\begin{aligned}
1+ & (\alpha \beta)^{1 / 4}+\{(1-\alpha)(1-\beta)\}^{1 / 4}+2^{4 / 3}\{\alpha \beta(1-\alpha)(1-\beta)\}^{1 / 12} \\
& =\left\{2\left(1+(\alpha \beta)^{1 / 2}+\{(1-\alpha)(1-\beta)\}^{1 / 2}\right)\right\}^{1 / 2} .
\end{aligned}
$$

We also need two of Ramanujan's modular equations of degree 3. The first is given by Lemma 3.3, while the second is given by [1, p. 231, Entry $5(\mathrm{ix})]$

$$
\{\alpha(1-\beta)\}^{1 / 2}+\{(1-\alpha) \beta\}^{1 / 2}=2\{\alpha \beta(1-\alpha)(1-\beta)\}^{1 / 8} .
$$

We shall apply (6.24) with $r=3$ and $p=23$. Thus, $\beta$ has degree 23 over $(1-\alpha)$. Thus, replacing $\alpha$ by $(1-\alpha)$, from (6.26) and (6.27), we find that, respectively,

$$
\{(1-\alpha) \beta\}^{1 / 8}+\{\alpha(1-\beta)\}^{1 / 8}+2^{2 / 3}\{\alpha \beta(1-\alpha)(1-\beta)\}^{1 / 24}=1
$$

and

$$
\begin{aligned}
1+ & \{(1-\alpha) \beta\}^{1 / 4}+\{\alpha(1-\beta)\}^{1 / 4}+2^{4 / 3}\{\alpha \beta(1-\alpha)(1-\beta)\}^{1 / 12} \\
& =\left\{2\left(1+\{(1-\alpha) \beta\}^{1 / 2}+\{\alpha(1-\beta)\}^{1 / 2}\right)\right\}^{1 / 2} .
\end{aligned}
$$

For brevity, in the remainder of the proof, set

$$
G=G_{69} \quad \text { and } \quad G^{\prime}=G_{23 / 3} .
$$

By (6.21), we can rewrite (6.29) in the form

$$
\{(1-\alpha) \beta\}^{1 / 8}+\{\alpha(1-\beta)\}^{1 / 8}=1-\sqrt{2}\left(G G^{\prime}\right)^{-1} .
$$

Setting $u=\left(G G^{\prime}\right)^{-1}$ and squaring both sides, we deduce that

$$
\{(1-\alpha) \beta\}^{1 / 4}+\{\alpha(1-\beta)\}^{1 / 4}=1+2 u^{2}-2 \sqrt{2} u-\sqrt{2} u^{3} .
$$

Substituting (6.31) into (6.30), we find that

$$
2+4 u^{2}-2 \sqrt{2} u-\sqrt{2} u^{3}=\sqrt{2}\left(1+\{(1-\alpha) \beta\}^{1 / 2}+\{\alpha(1-\beta)\}^{1 / 2}\right)^{1 / 2} .
$$

Then, using (6.28) in (6.32), we deduce that

$$
2+4 u^{2}-2 \sqrt{2} u-\sqrt{2} u^{3}=\sqrt{2}\left(1+\sqrt{2} u^{3}\right)^{1 / 2} .
$$

Squaring both sides and simplifying, we arrive at

$$
2-8 \sqrt{2} u+24 u^{2}-22 \sqrt{2} u^{3}+24 u^{4}-8 \sqrt{2} u^{5}+2 u^{6}=0,
$$


which, with $x=u+1 / u$, is equivalent to

$$
\begin{aligned}
22 \sqrt{2} & =2\left(u^{3}+u^{-3}\right)-8 \sqrt{2}\left(u^{2}+u^{-2}\right)+24\left(u+u^{-1}\right) \\
& =2\left(x^{3}-3 x\right)-8 \sqrt{2}\left(x^{2}-2\right)+24 x .
\end{aligned}
$$

Simplifying, we find that

$$
x^{3}-4 \sqrt{2} x^{2}+9 x-3 \sqrt{2}=0 .
$$

By inspection, we verify that $\sqrt{2}$ is a root. Now $G_{n}$ is a monotonically increasing function of $n$, and it is not difficult to numerically check that the root that we seek is greater than $\sqrt{2}$. Thus,

$$
x^{2}-3 \sqrt{2} x+3=0,
$$

and so $x=(3+\sqrt{3}) / \sqrt{2}$. Since $x=u+1 / u$, we find that

$$
\frac{1}{u}=\sqrt{\frac{6+3 \sqrt{3}}{4}}+\sqrt{\frac{2+3 \sqrt{3}}{4}}
$$

since $u<1$.

We now apply Lemma 3.3. Noting that $P=u^{3}$, we see that we want to calculate

$$
\begin{aligned}
u^{-3}-u^{3} & =\sqrt{\left(u^{-3}+u^{3}\right)^{2}-4} \\
& =\sqrt{\left(x^{3}-3 x\right)^{2}-4} \\
& =\sqrt{\left(\left(\frac{3+\sqrt{3}}{\sqrt{2}}\right)^{3}-3\left(\frac{3+\sqrt{3}}{\sqrt{2}}\right)\right)^{2}-4} \\
& =\sqrt{374+216 \sqrt{3}} .
\end{aligned}
$$

Thus, by Lemma 3.3,

$$
\left(\frac{G^{\prime}}{G}\right)^{6}+\left(\frac{G}{G^{\prime}}\right)^{6}=2 \sqrt{2}\left(u^{-3}-u^{3}\right)=2 \sqrt{2} \sqrt{374+216 \sqrt{3}}
$$

Solving for $G / G^{\prime}$, we deduce that

$$
\frac{G}{G^{\prime}}=(\sqrt{748+432 \sqrt{3}}+\sqrt{747+432 \sqrt{3}})^{1 / 6} .
$$

Thus, by (6.33) and (6.34),

$$
\begin{aligned}
G=\sqrt{\frac{G}{G^{\prime}}} u^{-1 / 2}= & (\sqrt{748+432 \sqrt{3}}+\sqrt{747+432 \sqrt{3}})^{1 / 12} \\
& \times\left(\sqrt{\frac{6+3 \sqrt{3}}{4}}+\sqrt{\frac{2+3 \sqrt{3}}{4}}\right)^{1 / 2} .
\end{aligned}
$$

To complete the proof, it suffices to show that

$$
(\sqrt{748+432 \sqrt{3}}+\sqrt{747+432 \sqrt{3}})^{2}=\left(\frac{5+\sqrt{23}}{\sqrt{2}}\right)^{2}\left(\frac{3 \sqrt{3}+\sqrt{23}}{2}\right)^{3},
$$

which is a straightforward task. 
Second Proof of Theorem 4.3. We need two of Ramanujan's modular equations of both degrees 7 and 11. If $\beta$ has degree 7 over $\alpha$, then [1, pp. 314, 315, Entry 19(i), (viii)]

$$
(\alpha \beta)^{1 / 8}+\{(1-\alpha)(1-\beta)\}^{1 / 8}=1
$$

and

$m-\frac{7}{m}=2\left((\alpha \beta)^{1 / 8}-\{(1-\alpha)(1-\beta)\}^{1 / 8}\right)\left(2+(\alpha \beta)^{1 / 4}+\{(1-\alpha)(1-\beta)\}^{1 / 4}\right)$,

where $m=\varphi^{2}(q) / \varphi^{2}\left(q^{7}\right)$. If $\beta$ has degree 11 over $\alpha$, then [1, p. 363, Entry $7(\mathrm{i})$, (ii)]

$$
(\alpha \beta)^{1 / 4}+\{(1-\alpha)(1-\beta)\}^{1 / 4}+2\{16 \alpha \beta(1-\alpha)(1-\beta)\}^{1 / 12}=1
$$

and

(6.38)

$$
m^{\prime}-\frac{11}{m^{\prime}}=2\left((\alpha \beta)^{1 / 4}-\{(1-\alpha)(1-\beta)\}^{1 / 4}\right)\left(4+(\alpha \beta)^{1 / 4}+\{(1-\alpha)(1-\beta)\}^{1 / 4}\right),
$$

where $m^{\prime}=\varphi^{2}(q) / \varphi^{2}\left(q^{11}\right)$.

If $q=\exp (-\pi \sqrt{11 / 7})$, by $(6.21)$,

$$
G_{11 / 7}=\{4 \alpha(1-\alpha)\}^{-1 / 24} \quad \text { and } \quad G_{77}=\{4 \beta(1-\beta)\}^{-1 / 24} .
$$

Thus, setting $u=\left(G_{77} G_{11 / 7}\right)^{-1}$, we deduce from (6.35) that

$$
\begin{aligned}
\left((\alpha \beta)^{1 / 8}-\{(1-\alpha)(1-\beta)\}^{1 / 8}\right)^{2}= & \left((\alpha \beta)^{1 / 8}+\{(1-\alpha)(1-\beta)\}^{1 / 8}\right)^{2} \\
& -4\{\alpha(1-\alpha) \beta(1-\beta)\}^{1 / 8} \\
= & 1-2 \sqrt{2} u^{3}
\end{aligned}
$$

and

$$
\begin{aligned}
(\alpha \beta)^{1 / 4}+\{(1-\alpha)(1-\beta)\}^{1 / 4}= & \left((\alpha \beta)^{1 / 8}+\{(1-\alpha)(1-\beta)\}^{1 / 8}\right)^{2} \\
& -2\{\alpha(1-\alpha) \beta(1-\beta)\}^{1 / 8} \\
= & 1-\sqrt{2} u^{3} .
\end{aligned}
$$

Thus, from (6.36),

$$
m-\frac{7}{m}=2\left(1-2 \sqrt{2} u^{3}\right)^{1 / 2}\left(3-\sqrt{2} u^{3}\right),
$$

where $m=\varphi^{2}\left(e^{-\pi \sqrt{11 / 7}}\right) / \varphi^{2}\left(e^{-\pi \sqrt{77}}\right)$.

Let $q=\exp (-\pi \sqrt{7 / 11})$, and note that $u=\left(G_{77} G_{11 / 7}\right)^{-1}=\left(G_{77} G_{7 / 11}\right)^{-1}$. Thus, by (6.37),

$$
(\alpha \beta)^{1 / 4}+\{(1-\alpha)(1-\beta)\}^{1 / 4}=1-2 u^{2}
$$

and

$$
\begin{aligned}
\left((\alpha \beta)^{1 / 4}-\{(1-\alpha)(1-\beta)\}^{1 / 4}\right)^{2}= & \left((\alpha \beta)^{1 / 4}+\{(1-\alpha)(1-\beta)\}^{1 / 4}\right)^{2} \\
& -4\{\alpha(1-\alpha) \beta(1-\beta)\}^{1 / 4} \\
= & \left(1-2 u^{2}\right)^{2}-2 u^{6}
\end{aligned}
$$


Hence, from (6.38),

$$
m^{\prime}-\frac{11}{m^{\prime}}=2\left(\left(1-2 u^{2}\right)^{2}-2 u^{6}\right)^{1 / 2}\left(5-2 u^{2}\right),
$$

where $m^{\prime}=\varphi^{2}\left(e^{-\pi \sqrt{7 / 11}}\right) / \varphi^{2}\left(e^{-\pi \sqrt{77}}\right)$.

From (6.25), we see that

$$
m^{\prime}=\sqrt{\frac{11}{7}} m
$$

Since

$$
m-\frac{7}{m}=\sqrt{\frac{7}{11}} m^{\prime}-\sqrt{\frac{11}{7}} \frac{7}{m^{\prime}}=\sqrt{\frac{7}{11}}\left(m^{\prime}-\frac{11}{m^{\prime}}\right),
$$

we deduce from (6.39) and (6.40) that

$$
2\left(1-2 \sqrt{2} u^{3}\right)^{1 / 2}\left(3-\sqrt{2} u^{3}\right)=\sqrt{\frac{7}{11}} 2\left(\left(1-2 u^{2}\right)^{2}-2 u^{6}\right)^{1 / 2}\left(5-2 u^{2}\right) .
$$

Squaring both sides and simplifying, we find that

$$
4 u^{10}-11 \sqrt{2} u^{9}-98 u^{8}+327 u^{6}-322 u^{4}-66 \sqrt{2} u^{3}+210 u^{2}-19=0 .
$$

Isolating the terms involving $\sqrt{2}$ on one side of the equation, squaring both sides, simplifying, and factoring, we deduce that

$$
\begin{aligned}
\left(u^{8}\right. & \left.-8 u^{6}+7 u^{4}-8 u^{2}+1\right) \\
& \times\left(196 u^{12}-1418 u^{10}+6044 u^{8}-13262 u^{6}+13073 u^{4}-5092 u^{2}+361\right)=0 .
\end{aligned}
$$

Now $x:=u^{2}$ is an algebraic integer (see Lemma 7.2) and so must be a root of a monic irreducible polynomial. The latter polynomial in (6.41) is irreducible, and so $x$ must be a root of the former polynomial in (6.41). Alternatively, we used Mathematica to numerically determine the roots of the latter polynomial and found that $u$ is not one of these roots. Thus,

$$
x^{4}-8 x^{3}+7 x^{2}-8 x+1=x^{2}\left((x+1 / x)^{2}-8(x+1 / x)+5\right)=0 .
$$

Since $x+1 / x>1$,

$$
x+\frac{1}{x}=4+\sqrt{11} .
$$

Thus,

$$
u+\frac{1}{u}=\sqrt{x+\frac{1}{x}+2}=\sqrt{6+\sqrt{11}} .
$$

Since $u<1$, we find that

$$
\frac{1}{u}=\sqrt{\frac{6+\sqrt{11}}{4}}+\sqrt{\frac{2+\sqrt{11}}{4}} .
$$


Lastly, we apply Lemma 3.5. Since $P=u^{-3}$, we deduce, by (6.42), that

$$
\begin{aligned}
Q+Q^{-1} & =2 \sqrt{2}\left(u^{-3}+u^{3}\right)-7 \\
& =2 \sqrt{2}\left(\left(u+u^{-1}\right)^{3}-3\left(u+u^{-1}\right)\right)-7 \\
& =2 \sqrt{2}(3+\sqrt{11}) \sqrt{6+\sqrt{11}}-7 \\
& =2(3+\sqrt{11})(1+\sqrt{11})-7 \\
& =21+8 \sqrt{11}
\end{aligned}
$$

Hence,

$$
\begin{aligned}
\frac{1}{Q} & =\frac{21+8 \sqrt{11}}{2}+\frac{\sqrt{1141+326 \sqrt{11}}}{2}=\frac{21}{2}+4 \sqrt{11}+\frac{\sqrt{7}}{2}(8+3 \sqrt{11}) \\
& =(8+3 \sqrt{7})\left(\frac{\sqrt{11}+\sqrt{7}}{2}\right) .
\end{aligned}
$$

In conclusion, by (6.42) and (6.43),

$$
\begin{aligned}
G_{77} & =Q^{-1 / 8} u^{-1 / 2} \\
& =(8+3 \sqrt{7})^{1 / 8}\left(\frac{\sqrt{11}+\sqrt{7}}{2}\right)^{1 / 8}\left(\sqrt{\frac{6+\sqrt{11}}{4}}+\sqrt{\frac{2+\sqrt{11}}{4}}\right)^{1 / 2},
\end{aligned}
$$

and the proof is complete.

Second Proof of Theorem 4.4. We need two of Ramanujan's modular equations, one of degree 3 and one of degree 47. If $\beta$ has degree 3 over $\alpha[1$, p. 231, Entry $5(\mathrm{ix})]$,

$$
\{\alpha(1-\beta)\}^{1 / 2}+\{\beta(1-\alpha)\}^{1 / 2}=2\{\alpha \beta(1-\alpha)(1-\beta)\}^{1 / 8} .
$$

If $\beta$ is of degree 47 over $\alpha$ [1, p. 444, Entry 23(i)],

$$
\begin{aligned}
2\left(\frac{1}{2}(1+(\right. & \left.\left.\alpha \beta)^{1 / 2}+\{(1-\alpha)(1-\beta)\}^{1 / 2}\right)\right)^{1 / 2} \\
= & 1+(\alpha \beta)^{1 / 4}+\{(1-\alpha)(1-\beta)\}^{1 / 4} \\
& +4^{1 / 3}\{\alpha \beta(1-\alpha)(1-\beta)\}^{1 / 24}\left(1+(\alpha \beta)^{1 / 8}+\{(1-\alpha)(1-\beta)\}^{1 / 8}\right) .
\end{aligned}
$$

Let $q=\exp (-\pi \sqrt{47 / 3})$. Then, by $(6.21)$,

$$
G^{\prime}:=G_{47 / 3}=\{4 \alpha(1-\alpha)\}^{-1 / 24} \quad \text { and } \quad G:=G_{141}=\{4 \beta(1-\beta)\}^{-1 / 24} \text {. }
$$

Applying (6.24) with $r=3$ and $p=47$, we find that $\beta$ has degree 47 over $(1-\alpha)$ when $\beta$ has degree 3 over $\alpha$. Thus, by (6.45),

$$
\begin{aligned}
2\left(\frac{1}{2}(1\right. & \left.\left.+\{(1-\alpha) \beta\}^{1 / 2}+\{\alpha(1-\beta)\}^{1 / 2}\right)\right)^{1 / 2} \\
(6.46)=1 & +\{(1-\alpha) \beta\}^{1 / 4}+\{\alpha(1-\beta)\}^{1 / 4} \\
& +4^{1 / 3}\{\alpha(1-\alpha) \beta(1-\beta)\}^{1 / 24}\left(1+\{(1-\alpha) \beta\}^{1 / 8}+\{\alpha(1-\beta)\}^{1 / 8}\right) .
\end{aligned}
$$


If $u:=\left(G G^{\prime}\right)^{-1}$, by $(6.44)$,

$$
\{\alpha(1-\beta)\}^{1 / 2}+\{\beta(1-\alpha)\}^{1 / 2}=\sqrt{2} u^{3} .
$$

Hence,

$$
\begin{aligned}
\left(\{\alpha(1-\beta)\}^{1 / 4}\right. & \left.+\{\beta(1-\alpha)\}^{1 / 4}\right)^{2} \\
& =\{\alpha(1-\beta)\}^{1 / 2}+\{\beta(1-\alpha)\}^{1 / 2}+2\{\alpha(1-\alpha) \beta(1-\beta)\}^{1 / 4} \\
& =\sqrt{2} u^{3}+u^{6}
\end{aligned}
$$

and

$$
\left(\{\alpha(1-\beta)\}^{1 / 8}+\{\beta(1-\alpha)\}^{1 / 8}\right)^{2}
$$

$$
\begin{aligned}
& =\{\alpha(1-\beta)\}^{1 / 4}+\{\beta(1-\alpha)\}^{1 / 4}+2\{\alpha(1-\alpha) \beta(1-\beta)\}^{1 / 8} \\
& =\left(\sqrt{2} u^{3}+u^{6}\right)^{1 / 2}+\sqrt{2} u^{3} .
\end{aligned}
$$

Substituting (6.47)-(6.49) into (6.46), we find that

$$
\begin{aligned}
& 2\left(\frac{1}{2}\left(1+\sqrt{2} u^{3}\right)\right)^{1 / 2} \\
& \quad=1+\left(\sqrt{2} u^{3}+u^{6}\right)^{1 / 2}+\sqrt{2} u\left(1+\left(\left(\sqrt{2} u^{3}+u^{6}\right)^{1 / 2}+\sqrt{2} u^{3}\right)^{1 / 2}\right) .
\end{aligned}
$$

Using Gröbner bases, A. Strzebonski denested (6.50) and obtained a polynomial of degree 48 for $u$. The value of $u$ that we seek is a root of the factor $u^{8}-32 u^{6}+$ $15 u^{4}-32 u^{2}+1$ of this 48 th degree polynomial. If $x=u^{2}$, then

$$
x^{4}-32 x^{3}+15 x^{2}-32 x+1=x^{2}\left((x+1 / x)^{2}-32(x+1 / x)+13\right)=0 .
$$

Since $x+1 / x>1$, we find that

$$
x+\frac{1}{x}=16+9 \sqrt{3}
$$

Hence,

$$
u+\frac{1}{u}=\sqrt{18+9 \sqrt{3}}
$$

so that

$$
\frac{1}{u}=\sqrt{\frac{18+9 \sqrt{3}}{4}}+\sqrt{\frac{14+9 \sqrt{3}}{4}} .
$$

Lastly, we apply Lemma 3.3 with $P=u^{3}$ and $Q=\left(G^{\prime} / G\right)^{6}$ to deduce, from (6.51), that

$$
\begin{aligned}
Q+\frac{1}{Q} & =2 \sqrt{2}\left(u^{-3}-u^{3}\right) \\
& =2 \sqrt{2}\left(\left(u^{-1}-u\right)^{3}+3\left(u^{-1}-u\right)\right) \\
& =2 \sqrt{2}(14+9 \sqrt{3})^{1 / 2}(17+9 \sqrt{3}) .
\end{aligned}
$$

Solving for $1 / Q$, we find that

$$
\frac{1}{Q}=\sqrt{2}(14+9 \sqrt{3})^{1 / 2}(17+9 \sqrt{3})+\sqrt{31419+18144 \sqrt{3}}
$$


Thus, by (6.51) and (6.52),

$$
\begin{aligned}
G_{141}=Q^{-1 / 12} u^{-1 / 2}= & \left(\sqrt{2}(14+9 \sqrt{3})^{1 / 2}(17+9 \sqrt{3})+\sqrt{31419+18144 \sqrt{3}}\right)^{1 / 12} \\
& \times\left(\sqrt{\frac{18+9 \sqrt{3}}{4}}+\sqrt{\frac{14+9 \sqrt{3}}{4}}\right)^{1 / 2} .
\end{aligned}
$$

It remains to show that

$$
\sqrt{2}(14+9 \sqrt{3})^{1 / 2}(17+9 \sqrt{3})+\sqrt{31419+18144 \sqrt{3}}=(4 \sqrt{3}+\sqrt{47})^{3 / 2}\left(\frac{7+\sqrt{47}}{\sqrt{2}}\right),
$$

which is easily accomplished via Mathematica.

Second Proof of Theorem 4.5. We need two modular equations, one of degree 5 and the other of degree 29. The first is found in Ramanujan's second notebook. If $\beta$ has degree 5 over $\alpha$, then [1, p. 281, Entry 13(x)]

$$
\{\alpha(1-\beta)\}^{1 / 4}+\{\beta(1-\alpha)\}^{1 / 4}=2^{2 / 3}\{\alpha \beta(1-\alpha)(1-\beta)\}^{1 / 24} .
$$

The second is found in Ramanujan's first notebook, but curiously not in his second. R. Russell [26] established this modular equation in 1890, but his formulation is imprecise; in particular, it has a sign ambiguity. We give Ramanujan's formulation. Let

$$
\begin{aligned}
& P=1-\sqrt{\alpha \beta}-\sqrt{(1-\alpha)(1-\beta)}, \\
& Q=64\{\sqrt{\alpha \beta}+\sqrt{(1-\alpha)(1-\beta)}-\sqrt{\alpha \beta(1-\alpha)(1-\beta)}\},
\end{aligned}
$$

and

$$
R=32 \sqrt{\alpha \beta(1-\alpha)(1-\beta)} .
$$

Then, if $\beta$ has degree 29 over $\alpha$,

$$
\sqrt{P}\left(P^{2}+17 P R^{1 / 3}-9 R^{2 / 3}\right)=R^{1 / 6}\left(9 P^{2}+Q-13 P R^{1 / 3}+15 R^{2 / 3}\right) .
$$

Let $q=\exp (-\pi \sqrt{29 / 5})$, so that we may apply (6.21) and (6.24) with $r=5$ and $p=29$. If $u=\left(G_{145} G_{29 / 5}\right)^{-1}$, then, by (6.53),

$$
\begin{aligned}
\{\alpha(1-\beta)\}^{1 / 2}+\{\beta(1-\alpha)\}^{1 / 2}= & \left(\{\alpha(1-\beta)\}^{1 / 4}+\{\beta(1-\alpha)\}^{1 / 4}\right)^{2} \\
& -2\{\alpha(1-\alpha) \beta(1-\beta)\}^{1 / 4} \\
= & 2 u^{2}-u^{6} .
\end{aligned}
$$

Thus, by (6.55), with $\alpha$ replaced by $(1-\alpha)$,

$$
\begin{aligned}
& P=1-2 u^{2}+u^{6}, \\
& Q=128 u^{2}-64 u^{6}-16 u^{12},
\end{aligned}
$$

and

$$
R=8 u^{12}
$$


Substitute these values into (6.54), square both sides, simplify, and factor, with the help of Mathematica. We then find that

$$
\begin{gathered}
\left(u^{2}+1\right)\left(u^{4}-u^{2}-1\right)\left(u^{4}-u^{2}+1\right)\left(u^{8}-20 u^{6}-43 u^{4}-20 u^{2}+1\right) \\
\times\left(u^{12}-9 u^{10}+181 u^{8}-126 u^{6}-181 u^{4}-9 u^{2}-1\right)=0 .
\end{gathered}
$$

In numerically checking the roots of each of these polynomials, we find that $x:=u^{2}$ is a root of

$$
x^{4}-20 x^{3}-43 x^{2}-20 x+1=x^{2}\left((x+1 / x)^{2}-20(x+1 / x)-45\right)=0 .
$$

Thus, $x+1 / x=10+\sqrt{145}$, and so $u-1 / u=\sqrt{8+\sqrt{145}}$. Hence,

$$
\frac{1}{u}=\sqrt{\frac{8+\sqrt{145}}{4}}+\sqrt{\frac{12+\sqrt{145}}{4}} .
$$

Lastly, we apply Lemma 3.4 with $P=u^{2}$ and $Q=\left(G_{29 / 5} / G_{145}\right)^{3}$. Then

$$
\begin{aligned}
Q+\frac{1}{Q}=2\left(\frac{1}{P}-P\right) & =2\left(\frac{1}{u}+u\right)\left(\frac{1}{u}-u\right) \\
& =2 \sqrt{12+\sqrt{145}} \sqrt{8+\sqrt{145}} \\
& =2 \sqrt{241+20 \sqrt{145}} .
\end{aligned}
$$

Hence,

$$
\frac{1}{Q}=\sqrt{241+20 \sqrt{145}}+\sqrt{240+20 \sqrt{145}}
$$

From (6.56) and (6.57),

$$
\begin{aligned}
G_{145}=Q^{-1 / 6} u^{-1 / 2}= & (\sqrt{241+20 \sqrt{145}}+\sqrt{240+20 \sqrt{145}})^{1 / 6} \\
& \times\left(\sqrt{\frac{12+\sqrt{145}}{4}}+\sqrt{\frac{8+\sqrt{145}}{4}}\right)^{1 / 2} .
\end{aligned}
$$

To complete the proof, we must show that

$$
(\sqrt{5}+2)\left(\frac{\sqrt{29}+5}{2}\right)=\left(\sqrt{\frac{12+\sqrt{145}}{4}}+\sqrt{\frac{8+\sqrt{145}}{4}}\right)^{2}
$$

and

$$
\left(\sqrt{\frac{17+\sqrt{145}}{8}}+\sqrt{\frac{9+\sqrt{145}}{8}}\right)^{3}=\sqrt{241+20 \sqrt{145}}+\sqrt{240+20 \sqrt{145}} .
$$

Both equalities are easily verified.

Second Proof of Theorem 4.7. In addition to the modular equation of degree 3 given by (6.44), we need Ramanujan's modular equation of degree 71. If $\beta$ has 
degree 71 over $\alpha$, then [1, p. 444, Entry 23(ii)]

$$
\begin{aligned}
1+(\alpha \beta)^{1 / 4}+ & \{(1-\alpha)(1-\beta)\}^{1 / 4}-\left(\frac{1}{2}\left(1+(\alpha \beta)^{1 / 2}+\{(1-\alpha)(1-\beta)\}^{1 / 2}\right)\right)^{1 / 2} \\
= & (\alpha \beta)^{1 / 8}+\{(1-\alpha)(1-\beta)\}^{1 / 8}-\{\alpha \beta(1-\alpha)(1-\beta)\}^{1 / 8} \\
& +2^{2 / 3}\{\alpha \beta(1-\alpha)(1-\beta)\}^{1 / 24}\left(1-(\alpha \beta)^{1 / 8}-\{(1-\alpha)(1-\beta)\}^{1 / 8}\right) .
\end{aligned}
$$

Let $r=3$ and $p=71$ in equalities (6.21) and principle (6.24). Thus, $\beta$ has degree 71 over $(1-\alpha)$. Replacing $\alpha$ by $1-\alpha$ in (6.58) and employing (6.47)-(6.49), but now with $u=\left(G_{213} G_{71 / 3}\right)^{-1}$, we deduce that

$$
\begin{aligned}
1+ & \left(\sqrt{2} u^{3}+u^{6}\right)^{1 / 2}-\left(\frac{1}{2}\left(1+\sqrt{2} u^{3}\right)\right)^{1 / 2} \\
= & \left(\left(\sqrt{2} u^{3}+u^{6}\right)^{1 / 2}+\sqrt{2} u^{3}\right)^{1 / 2} \\
& -\frac{1}{\sqrt{2}} u^{3}+\sqrt{2} u\left(1-\left(\left(\sqrt{2} u^{3}+u^{6}\right)^{1 / 2}+\sqrt{2} u^{3}\right)^{1 / 2}\right) \\
= & \left(\left(\sqrt{2} u^{3}+u^{6}\right)^{1 / 2}+\sqrt{2} u^{3}\right)^{1 / 2}(1-\sqrt{2} u)-\frac{1}{\sqrt{2}} u^{3}+\sqrt{2} u .
\end{aligned}
$$

Using resultants, Strzebonski and M. Trott denested (6.59) and found a polynomial that factors into several polynomials of degrees 8, 12, and 28. Numerically eliminating all factors except one, we find that $u$ satisfies

$$
u^{8}-80 u^{6}-126 u^{4}-80 u^{2}+1=0 .
$$

Letting $u^{2}=: x$, and solving for $x+1 / x$, we find that $x+1 / x=40+24 \sqrt{3}$. It then follows that $u+1 / u=\sqrt{42+24 \sqrt{3}}$. Hence,

$$
\frac{1}{u}=\sqrt{\frac{21+12 \sqrt{3}}{2}}+\sqrt{\frac{19+12 \sqrt{3}}{2}} .
$$

Lastly, we invoke Lemma 3.3 with $P=\left(G_{213} G_{71 / 3}\right)^{-3}=u^{3}$ and $Q=\left(G_{71 / 3} / G_{213}\right)^{6}$. So, by Lemma 3.3 and (6.60),

$$
Q+\frac{1}{Q}=2 \sqrt{2}\left(u^{-3}-u^{3}\right)=4(19+12 \sqrt{3})^{1 / 2}(41+24 \sqrt{3}) .
$$

Solving for $1 / Q$, we find that

$$
\frac{1}{Q}=2(19+12 \sqrt{3})^{1 / 2}(41+24 \sqrt{3})+\sqrt{542,475+313,200 \sqrt{3}} .
$$


Hence, by (6.60) and (6.61),

$$
\begin{aligned}
G_{213}= & Q^{-1 / 12} u^{-1 / 2} \\
= & \left(2(19+12 \sqrt{3})^{1 / 2}(41+24 \sqrt{3})+\sqrt{542,475+313,200 \sqrt{3}}\right)^{1 / 12} \\
& \times\left(\sqrt{\frac{21+12 \sqrt{3}}{2}}+\sqrt{\frac{19+12 \sqrt{3}}{2}}\right)^{1 / 2} .
\end{aligned}
$$

It thus remains to show that

$$
\begin{aligned}
2(19+12 \sqrt{3})^{1 / 2}(41+24 \sqrt{3})+ & \sqrt{542,475+313,200 \sqrt{3}} \\
= & \left(\frac{5 \sqrt{3}+\sqrt{71}}{2}\right)^{3 / 2}\left(\frac{59+7 \sqrt{71}}{\sqrt{2}}\right),
\end{aligned}
$$

which can be verified via Mathematica.

\section{Watson's Empirical Process}

In [30], Watson employed an "empirical process" to evaluate 14 of Ramanujan's class invariants. Motivated by Watson's idea, we succeeded in formulating theorems which give rigorous evaluations of $G_{p q}$ and $G_{p / q}$ when $p$ and $q$ are distinct primes satisfying $p q \equiv 1(\bmod 4)$ and $h(\sqrt{-p q})=8$.

Let $K=\mathbb{Q}(\sqrt{-m})$ ( $m$ squarefree) be an imaginary quadratic field, and let $\mathfrak{O}_{K}$ be its ring of integers. By class field theory [13, p. 191, Theorem 13.1], there exists an everywhere unramified extension $K^{(1)} \mid K$ such that the Galois group

$$
\operatorname{Gal}\left(K^{(1)} \mid K\right) \simeq C_{K}
$$

The field $K^{(1)}$ is known as the Hilbert class field of $K$. A Hilbert class field of $K$ is usually defined as the maximal unramified abelian extension of $K$.

Let $\mathfrak{a}=\left[\tau_{1}, \tau_{2}\right]$ be an $\mathfrak{O}_{K}$-ideal. Define

$$
j(\mathfrak{a})=j\left(\left[\tau_{1}, \tau_{2}\right]\right)=1728 \frac{g_{2}^{3}\left(\left[\tau_{1}, \tau_{2}\right]\right)}{g_{2}^{3}\left(\left[\tau_{1}, \tau_{2}\right]\right)-27 g_{3}^{2}\left(\left[\tau_{1}, \tau_{2}\right]\right)},
$$

where

$$
g_{2}\left(\left[\tau_{1}, \tau_{2}\right]\right)=60 \sum_{\substack{m, n=-\infty \\(m, n) \neq(0,0)}}^{\infty} \frac{1}{\left(m \tau_{1}+n \tau_{2}\right)^{4}}
$$

and

$$
g_{3}\left(\left[\tau_{1}, \tau_{2}\right]\right)=140 \sum_{\substack{m, n=-\infty \\(m, n) \neq(0,0)}}^{\infty} \frac{1}{\left(m \tau_{1}+n \tau_{2}\right)^{6}} .
$$

It is clear from the definitions of $g_{2}\left(\left[\tau_{1}, \tau_{2}\right]\right)$ and $g_{3}\left(\left[\tau_{1}, \tau_{2}\right]\right)$ that

$$
j\left(\left[\tau_{1}, \tau_{2}\right]\right)=j([1, \tau])=: j(\tau),
$$

where $\tau=\tau_{2} / \tau_{1}$. We will also let

$$
\gamma_{2}(\tau)=\sqrt[3]{j(\tau)}
$$

with the cube root being real-valued when $j(\mathfrak{a})$ is real. 
It is well known that $K^{(1)}=K\left(j\left(\mathfrak{O}_{K}\right)\right)\left[11\right.$, p. 220 , Theorem 11.1]. If $D_{K}$ is the discriminant of $K$ and $3 \nmid D_{K}$, then $K^{(1)}=K\left(\gamma_{2}\left(\tau_{K}\right)\right)$ [11, p. 249, Theorem 12.2], where

$$
\tau_{K}=\left\{\begin{array}{lll}
\sqrt{-m}, & D_{K} \equiv 0 & (\bmod 4) \\
\frac{3+\sqrt{-m}}{2}, & D_{K} \equiv 1 & (\bmod 4)
\end{array}\right.
$$

Lemma 7.1. Let $\mathfrak{a}$ and $\mathfrak{b}$ be two $\mathfrak{O}_{K}$-ideals. Define $\sigma_{\mathfrak{a}}(j(\mathfrak{b}))$ by

$$
\sigma_{\mathfrak{a}}(j(\mathfrak{b}))=j(\overline{\mathfrak{a}} \mathfrak{b}),
$$

where $\mathfrak{a} \overline{\mathfrak{a}}$ is a principal ideal. Then $\sigma_{\mathfrak{a}}$ is a well-defined element of $\operatorname{Gal}\left(K^{(1)} \mid K\right)$, and $\mathfrak{a} \mapsto \sigma_{\mathfrak{a}}$ induces an isomorphism

$$
C_{K} \longrightarrow \operatorname{Gal}\left(K^{(1)} \mid K\right)
$$

Proof. See [11, p. 240, Corollary 11.37].

Lemma 7.2. Let $K=\mathbb{Q}(\sqrt{-p q})$, where $p$ and $q$ are two distinct primes satisfying $p q \equiv 1(\bmod 4)$, and let

$$
\gamma=\left\{\begin{array}{l}
4, \quad \text { if } 3 \nmid p q, \\
12, \quad \text { if } 3 \mid p q .
\end{array}\right.
$$

Then $G_{p q}^{\gamma}$ is a real unit generating the field $K^{(1)}$.

Proof. From [5, p. 290], we find that $G_{p q}^{12}$ is a real unit of $K^{(1)}$. Since [11, p. 257, Theorem 12.17]

$$
j\left(\mathfrak{O}_{K}\right)=j(\sqrt{-p q})=\frac{\left(16 G_{p q}^{24}-4\right)^{3}}{G_{p q}^{24}},
$$

we conclude that

$$
K^{(1)}=K\left(G_{p q}^{12}\right)
$$

Next, suppose that $3 \nmid p q$. Then $3 \nmid D_{K}$ and $\gamma_{2}\left(\tau_{K}\right)$ generates $K^{(1)}$. From the equality [11, p. 257, Theorem 12.17]

$$
\gamma_{2}(\sqrt{-p q})=\frac{16 G_{p q}^{24}-4}{G_{p q}^{8}}
$$

and (7.3), we find that $G_{p q}^{8} \in K^{(1)}$. Hence, $G_{p q}^{4} \in K^{(1)}$, by (7.3).

Remarks. In [5, p. 290], B. J. Birch quoted M. Deuring's results [12, p. 43] and indicated that $G_{p q}$ is a unit when $p q \equiv 1(\bmod 4)$. A more elaborate proof of this statement can be found in [9, Corollary 5.2]. In fact, from the treatment given in [9], one can show that $G_{p / q}$ is also a unit. This fact will be needed in our main theorem.

From class field theory, we know that if $H$ is a subgroup of $C_{K}$, then there exists an abelian and everywhere unramified extension $L \mid K$ such that

$$
\operatorname{Gal}\left(K^{(1)} \mid L\right) \simeq H
$$

In particular, when $H=C_{K}^{2}:=$ the subgroup of squares in $C_{K}$, the corresponding field $M \mid K$ is known as the genus field of $K$. One can show that $M$ is the maximal unramified extension of $K$ which is abelian over $\mathbb{Q}[11$, p. 122]. 
Theorem 7.3. Let $K$ and $\gamma$ be defined as in Lemma 7.2. If the order of $C_{K}$ is 8, then

$$
\alpha_{p, q}:=\left(G_{p q} G_{p / q}\right)^{\gamma}+\left(G_{p q} G_{p / q}\right)^{-\gamma}
$$

and

$$
\beta_{p, q}:=\left(\frac{G_{p q}}{G_{p / q}}\right)^{\gamma}+\left(\frac{G_{p q}}{G_{p / q}}\right)^{-\gamma}
$$

are algebraic integers which belong to the real quadratic field $R$, where $R \in\{\mathbb{Q}(\sqrt{p})$, $\mathbb{Q}(\sqrt{q}), \mathbb{Q}(\sqrt{p q})\}$, and where $R$ is a field such that none of the prime ideals $(2),(p)$, or $(q)$ are inert.

Proof. From the hypothesis, we deduce that $\mathfrak{a}_{1}=[1, \sqrt{-p q}], \mathfrak{a}_{2}=[q, \sqrt{-p q}]$, $\mathfrak{a}_{3}=[2,1+\sqrt{-p q}]$, and $\mathfrak{a}_{4}=[2 q, q+\sqrt{-p q}]$ are $\mathfrak{O}_{K}$-ideals lying in distinct equivalence classes (see Section 2). This implies that $C_{K}$ contains the Klein-four group generated by the ideal classes $\left[\mathfrak{a}_{i}\right]$ and $\left[\mathfrak{a}_{j}\right]$ for $i>j>1$. Using the isomorphism described in Lemma 7.1, we conclude that $\operatorname{Gal}\left(K^{(1)} \mid K\right)$ contains a Klein fourgroup $V$ generated by $\sigma_{\mathfrak{a}_{i}}$ and $\sigma_{\mathfrak{a}_{j}}$ for $i>j>1$. To show that $\alpha_{p, q}$ and $\beta_{p, q}$ belong to a field with degree 2 over $K$, it suffices to show that $\sigma_{\mathfrak{a}_{i}}$ and $\sigma_{\mathfrak{a}_{j}}$ fix $\alpha_{p, q}$ and $\beta_{p, q}$. More precisely, if $F:=F i x(V)$ is the field fixed by $V$, then by Galois theory $\left[25\right.$, p. 49, Theorem 63], $|F: K|=\left|\operatorname{Gal}\left(K^{(1)} \mid K\right): V\right|=2$ (since $\left|C_{K}\right|=8$ ), which implies that $F$ is of degree 2 over $K$. Since $\alpha_{p, q}$ and $\beta_{p, q}$ are real numbers in $F$, they belong to $R:=F \cap \mathbb{R}$, and $R$ is clearly a real quadratic field over $\mathbb{Q}$. The fact that they are algebraic integers follows from the fact that $G_{p q}^{\gamma}$ and $G_{p / q}^{\gamma}$ are units (see Lemma 7.2).

At this stage, we will assume that $3 \mid p q$. From [11, p. 257, Theorem 12.17], we have

$$
j\left(\mathfrak{a}_{2}\right)=j(\sqrt{-p / q})=\frac{\left(16 G_{p / q}^{24}-4\right)^{3}}{G_{p / q}^{24}} .
$$

By Lemma 7.1, we find that

$$
\sigma_{\mathfrak{a}_{2}}\left(j\left(\mathfrak{a}_{1}\right)\right)=j\left(\mathfrak{a}_{2} \mathfrak{a}_{1}\right)=j\left(\mathfrak{a}_{2}\right) .
$$

From (7.2), (7.5), and (7.6), we find that

$$
\frac{\left(16 \sigma_{\mathfrak{a}_{2}}^{2}\left(G_{p q}^{12}\right)-4\right)^{3}}{\sigma_{\mathfrak{a}_{2}}^{2}\left(G_{p q}^{12}\right)}=\frac{\left(16 G_{p / q}^{24}-4\right)^{3}}{G_{p / q}^{24}} .
$$

Simplifying (7.7), we deduce that

$$
(a-b)(a+b)\left\{64\left(a^{2}+b^{2}\right) a^{2} b^{2}-48 a^{2} b^{2}+1\right\}=0,
$$

where $a=\sigma_{\mathfrak{a}_{2}}\left(G_{p q}^{12}\right)$ and $b=G_{p / q}^{12}$. But

$$
64\left(a^{2}+b^{2}\right) a^{2} b^{2}-48 a^{2} b^{2}+1 \neq 0,
$$

for otherwise it would contradict the fact that $a$ and $b$ are algebraic integers. Thus, we deduce that

$$
\sigma_{\mathfrak{a}_{2}}\left(G_{p q}^{12}\right)= \pm G_{p / q}^{12}
$$

Similarly, corresponding to (7.8), we have

$$
\sigma_{\mathfrak{a}_{2}}\left(G_{p / q}^{12}\right)= \pm G_{p q}^{12} \quad \text { or } \quad \sigma_{\mathfrak{a}_{2}}\left(G_{p / q}^{12}\right)=\mp G_{p q}^{12}
$$


i.e., $\sigma_{\mathfrak{a}_{2}}\left(G_{p / q}^{12}\right)$ may have the same or opposite sign as $\sigma_{\mathfrak{a}_{2}}\left(G_{p q}^{12}\right)$. Since $\sigma_{\mathfrak{a}_{2}}^{2}=1$, the latter is inadmissible. Hence,

$$
\sigma_{\mathfrak{a}_{2}}\left(G_{p / q}^{12}\right)= \pm G_{p q}^{12}
$$

From (7.8) and (7.9), it is now clear that

$$
\sigma_{\mathfrak{a}_{2}}\left(\alpha_{p, q}\right)=\alpha_{p, q}
$$

and

$$
\sigma_{\mathfrak{a}_{2}}\left(\beta_{p, q}\right)=\beta_{p, q} .
$$

Next, by [11, p. 263], we find that

$$
j\left(\mathfrak{a}_{3}\right)=j\left(\frac{3+\sqrt{-p q}}{2}\right)=G_{p q}^{24}\left(\frac{16}{G_{p q}^{24}}-4\right)^{3}
$$

and

$$
j\left(\mathfrak{a}_{4}\right)=j\left(\frac{3+\sqrt{-p / q}}{2}\right)=G_{p / q}^{24}\left(\frac{16}{G_{p / q}^{24}}-4\right)^{3} .
$$

Now, applying Lemma 7.1 again, we have

$$
\sigma_{\mathfrak{a}_{3}}\left(j\left(\mathfrak{a}_{1}\right)\right)=j\left(\mathfrak{a}_{3}\right) .
$$

By (7.10) and (7.2), we find that

$$
\frac{\left(16 \sigma_{\mathfrak{a}_{3}}^{2}\left(G_{p q}^{12}\right)-4\right)^{3}}{\sigma_{\mathfrak{a}_{3}}^{2}\left(G_{p q}^{12}\right)}=G_{p q}^{24}\left(\frac{16}{G_{p q}^{24}}-4\right)^{3},
$$

which implies that

$$
\sigma_{\mathfrak{a}_{3}}\left(G_{p q}^{12}\right)= \pm G_{p q}^{-12}
$$

Similarly, since $\mathfrak{a}_{3} \mathfrak{a}_{2}$ is equivalent to $\mathfrak{a}_{4}$, we have

$$
\sigma_{\mathfrak{a}_{3}}\left(G_{p / q}^{12}\right)= \pm G_{p / q}^{-12} \quad \text { or } \quad \mp G_{p / q}^{-12}
$$

by $(7.11)$ and $(7.5)$, i.e., $\sigma_{\mathfrak{a}_{3}}\left(G_{p / q}^{12}\right)$ may have the same or opposite $\operatorname{sign}$ as $\sigma_{\mathfrak{a}_{3}}\left(G_{p q}^{12}\right)$. We will show that the latter case is inadmissible. If

$$
\sigma_{\mathfrak{a}_{2}}\left(G_{p / q}^{12}\right)= \pm G_{p q}^{12} \quad \text { and } \quad \sigma_{\mathfrak{a}_{3}}\left(G_{p / q}^{12}\right)=\mp G_{p / q}^{-12},
$$

then

$$
\sigma_{\mathfrak{a}_{2}} \sigma_{\mathfrak{a}_{3}}\left(G_{p q}^{12}\right)= \pm G_{p / q}^{-12} \quad \text { and } \quad \sigma_{\mathfrak{a}_{3}} \sigma_{\mathfrak{a}_{2}}\left(G_{p q}^{12}\right)=\mp G_{p / q}^{-12} .
$$

This is clearly a contradiction since $\sigma_{\mathfrak{a}_{2}} \sigma_{\mathfrak{a}_{3}}=\sigma_{\mathfrak{a}_{3}} \sigma_{\mathfrak{a}_{2}}$. Hence,

$$
\sigma_{\mathfrak{a}_{3}}\left(\alpha_{p, q}\right)=\alpha_{p, q}
$$

and

$$
\sigma_{\mathfrak{a}_{3}}\left(\beta_{p, q}\right)=\beta_{p, q} .
$$

Collecting our results, we see that both $\sigma_{\mathfrak{a}_{2}}$ and $\sigma_{\mathfrak{a}_{3}}$ fix $\alpha_{p, q}$ and $\beta_{p, q}$, and this implies that $\alpha_{p, q}$ and $\beta_{p, q}$ are real quadratic algebraic integers.

The proof for the case when $3 \nmid p q$ is similar. In this case, $G_{p q}^{4}$ generates $K^{(1)}$, and so $\sigma_{\mathfrak{a}_{i}}\left(G_{p q}^{4}\right)$ is well defined for $i>1$. Hence, we may deduce from (7.7) that

$$
16\left(\sigma_{\mathfrak{a}_{2}}\left(G_{p q}^{8}\right)\right)^{2}-\frac{4}{\sigma_{\mathfrak{a}_{2}}\left(G_{p q}^{8}\right)}=16 G_{p / q}^{16}-\frac{4}{G_{p / q}^{8}} .
$$


Simplifying (7.12), we have

$$
(a-b)\left(4 a^{2} b+4 a b^{2}+1\right)=0,
$$

where $a=\sigma_{\mathfrak{a}_{2}}\left(G_{p q}^{8}\right)$ and $b=G_{p / q}^{8}$. But

$$
4 a^{2} b+4 a b^{2}+1 \neq 0,
$$

for otherwise it would contradict the fact that $a$ and $b$ are algebraic integers. Hence, we deduce that

$$
\sigma_{\mathfrak{a}_{2}}\left(G_{p q}^{8}\right)=G_{p / q}^{8} .
$$

Now, since $\sigma_{\mathfrak{a}_{2}} \in \operatorname{Gal}\left(K^{(1)} \mid K\right)$ and $G_{p q}^{4}$ generates $K^{(1)}$ (see Lemma 7.2), we have

$$
\sigma_{\mathfrak{a}_{2}}\left(G_{p q}^{4}\right)= \pm G_{p / q}^{4} .
$$

The rest of the arguments are similar to those of the previous case, and we shall omit them.

We have already seen that $\alpha_{p, q}$ and $\beta_{p, q}$ lie in a real quadratic field $R$. Our next task is to give a necessary condition for $R$. First, we observe that $R=F \cap \mathbb{R}$, where $F=F i x(V)$ is an abelian, everywhere unramified extension of $K$ (see the paragraph before the statement of Theorem 7.3). Hence, $R \in\{\mathbb{Q}(\sqrt{p}), \mathbb{Q}(\sqrt{q}), \mathbb{Q}(\sqrt{p q})\}$. Next, we will show that none of the prime ideals $(2),(p)$ or $(q)$ are inert in $R$. Suppose the contrary holds. Then without loss of generality, we may assume that $(p)$ is inert in $R$. This implies that $\mathfrak{p}$ in $K$ is inert in $F$, where $\mathfrak{p} \mid(p)$ and the Frobenius automorphism $\left[\frac{F \mid K}{\mathfrak{p}}\right]$ has order 2 (see [11, pp. 106-107] or [13, pp. 126-127]).

On the other hand, we know that the Frobenius automorphism $\sigma_{\mathfrak{p}}=\left[\frac{K^{(1)} \mid K}{\mathfrak{p}}\right]$ has order 2 and that $\left.\left[\frac{K^{(1)} \mid K}{\mathfrak{p}}\right]\right|_{E}=1$, where

$$
E=F i x\left(\left[\frac{K^{(1)} \mid K}{\mathfrak{p}}\right]\right) \text {. }
$$

Since [13, p. 127, Property 2.3]

$$
\left[\frac{E \mid K}{\mathfrak{p}}\right]=\left.\left[\frac{K^{(1)} \mid K}{\mathfrak{p}}\right]\right|_{E},
$$

we find that $\left[\frac{E \mid K}{\mathfrak{p}}\right]$ has order 1. Consequently,

$$
\left[\frac{F \mid K}{\mathfrak{p}}\right]=\left.\left[\frac{E \mid K}{\mathfrak{p}}\right]\right|_{F}=1 .
$$

This clearly contradicts the last statement of the previous paragraph. Thus, $(p)$ is not inert in $R$.

Our next step is to determine $\alpha_{p, q}$ and $\beta_{p, q}$ using the numerical values of $G_{p q}$ and $G_{p / q}$. To achieve this, we need the following result.

Theorem 7.4. Let $R=\mathbb{Q}(\sqrt{m})$ be the field which contains $\alpha_{p, q}$ and $\beta_{p, q}$. If

$$
2 \alpha_{p, q}=a_{1}+a_{2} \sqrt{m}
$$


and

$$
2 \beta_{p, q}=b_{1}+b_{2} \sqrt{m}
$$

then $a_{1}, a_{2}, b_{1}$, and $b_{2}$ are positive integers.

Proof. Let $[\mathfrak{a}] \in \mathbf{A}:=\left\{\left[\mathfrak{a}_{2}\right],\left[\mathfrak{a}_{3}\right],\left[\mathfrak{a}_{4}\right]\right\}$, and let $H$ be the group generated by $[\mathfrak{a}]$. From the paragraph before the statement of Theorem 7.3, we know that there exists an abelian and everywhere unramified extension $L \mid K$ such that

$$
\operatorname{Gal}\left(K^{(1)} \mid L\right) \simeq H .
$$

In fact, from the isomorphism of Lemma 7.1, we find that $L=F i x\left(\sigma_{\mathfrak{a}}\right)$. Since $\operatorname{Gal}\left(K^{(1)} \mid K\right) \simeq \mathbb{Z}_{2} \oplus \mathbb{Z}_{4}$, the group

$$
\operatorname{Gal}(L \mid K) \simeq \mathbb{Z}_{2} \oplus \mathbb{Z}_{2} \quad \text { or } \quad \mathbb{Z}_{4} .
$$

The first case can only happen for exactly one element in $\mathbf{A}$, and the field $L$ in this case is the genus field $M$ of $K$. As for the second case, $\operatorname{Gal}(L \mid \mathbb{Q}) \simeq D_{8}$, the dihedral group of eight elements, since $L$ is generalized dihedral over $\mathbb{Q}$ [11, p. 191]. Hence, $\operatorname{Gal}(L \mid \mathbb{Q})$ is non-abelian.

Now, rewrite (7.13) as

$$
2\left(\eta+\eta^{-1}\right)=a_{1}+a_{2} \sqrt{m},
$$

where $\eta=\left(G_{p q} G_{p / q}\right)^{\gamma}$. Note that $\sigma_{\mathfrak{a}_{2}}$ fixes $\eta$ and $\sigma_{\mathfrak{a}_{3}}(\eta)=\eta^{-1}$. Therefore, the field $L:=K(\eta)=$ Fix $\left(\left\langle\sigma_{\mathfrak{a}_{2}}\right\rangle\right)$ is of degree 4 over $K$.

Suppose $L$ is the genus field of $K$. Since $\left.\sigma_{\mathfrak{a}_{2}}\right|_{L}=1$, we conclude that the ideal $\left[\mathfrak{a}_{2}\right]$ lies in an ideal class belonging to the principal genus. Hence, by Theorem 3.2, we may write

$$
\eta=\prod_{\chi\left(\mathbf{G}_{1}\right)=-1} \epsilon_{1}^{e_{1}}
$$

where

$$
e_{1}= \begin{cases}w h_{1} h_{2} / w_{2}, & \text { if } 3 \nmid p q, \\ 3 w h_{1} h_{2} / w_{2}, & \text { if } 3 \mid p q .\end{cases}
$$

Since $w=2$ and

$$
w_{2}= \begin{cases}2 \text { or } 4, & \text { if } 3 \nmid p q, \\ 2,4 \text { or } 6, & \text { if } 3 \mid p q,\end{cases}
$$

we conclude that $e_{1}$ must be of the form $e_{1}^{\prime} / 2$, where $e_{1}^{\prime} \in \mathbb{N}$. Hence, we may rewrite (7.16) as

$$
\eta=\prod_{\chi\left(\mathbf{G}_{1}\right)=-1} \epsilon_{1}^{e_{1}^{\prime} / 2} .
$$

Now, it is known that a fundamental unit of a real quadratic field takes the form $u+v \sqrt{d}$ with $u, v>0$ [6, p. 133]. Furthermore, if $\sqrt{u+v \sqrt{d}}=u^{\prime} \sqrt{d_{1}}+v^{\prime} \sqrt{d_{2}}$, then $u^{\prime}, v^{\prime} \geq 0$. Collecting these observations, we deduce that $\eta$ is of the form $u_{1}+u_{2} \sqrt{p}+u_{3} \sqrt{q}+u_{4} \sqrt{p q}$, where $u_{i} \geq 0$ for each $i$. Using (7.15) and (7.17), we conclude that $a_{1}$ and $a_{2}$ are positive integers.

Next, suppose $L$ is not the genus field. Then from the beginning of our discussion, $\operatorname{Gal}(L \mid \mathbb{Q}) \simeq D_{8}$ is non-abelian. We claim that there exists an element $\sigma$ in $\operatorname{Gal}(L \mid K)$ such that $\sigma(\eta)$ is complex. Suppose the contrary holds. Then $L \cap \mathbb{R}=\mathbb{Q}(\eta)$ would 
be Galois over $\mathbb{Q}$, and hence $\operatorname{Gal}(L \mid \mathbb{Q}(\eta))$ is a normal subgroup of $\operatorname{Gal}(L \mid \mathbb{Q})$. On the other hand, $\operatorname{Gal}(\mathbb{Q}(\eta) \mid \mathbb{Q}) \simeq \operatorname{Gal}(L \mid K)$, a normal subgroup of $\operatorname{Gal}(L \mid \mathbb{Q})[11$, p. 191]. Hence, $\operatorname{Gal}(L \mid \mathbb{Q})$ is isomorphic to the direct sum of $\operatorname{Gal}(L \mid \mathbb{Q}(\eta))$ and $\operatorname{Gal}(\mathbb{Q}(\eta) \mid \mathbb{Q})$ and is therefore an abelian group, and this contradicts our initial assumption.

Next, we will show that $\sigma(\sqrt{m})=-\sqrt{m}$. Suppose that the contrary holds. Then

$$
\sigma(\eta)+\sigma(\eta)^{-1}=\eta+\eta^{-1}
$$

and therefore

$$
\sigma(\eta)=\eta \quad \text { or } \quad \eta^{-1}
$$

This shows that $\sigma(\eta)$ is real, which contradicts our choice of $\sigma$. Now, applying $\sigma$ to $(7.15)$, we deduce that

$$
2\left(\sigma(\eta)+\sigma(\eta)^{-1}\right)=a_{1}-a_{2} \sqrt{m} .
$$

From $(7.15),(7.18)$, and the fact that $\sigma(\eta)$ is complex, we find that

$$
\left(a_{1}+a_{2} \sqrt{m}\right)^{2} \geq 16
$$

and

$$
\left(a_{1}-a_{2} \sqrt{m}\right)^{2}<16 .
$$

This implies that $4 a_{1} a_{2} \sqrt{m}>0$. Since $\eta>0$, we deduce that $a_{1}$ and $a_{2}$ are positive. The integrality of $a_{1}$ and $a_{2}$ follows easily from Theorem 7.3. In a similar way, we can show that $b_{1}$ and $b_{2}$ are positive integers in (7.14).

Remark. The argument given here for the case when $\operatorname{Gal}(L \mid \mathbb{Q})$ is non-abelian is due to H. Weber [11, p. 269].

Let $\mathfrak{R}_{K}$ be the subset of $\{\mathbb{Q}(\sqrt{p}), \mathbb{Q}(\sqrt{q}), \mathbb{Q}(\sqrt{p q})\}$ satisfying the last statement in Theorem 7.3. Note that, since $\left|\mathfrak{R}_{K}\right|$ is finite and $2 \alpha_{p, q}$ and $2 \beta_{p, q}$ lie in a discrete subset of the ring $\mathbb{Z}(\sqrt{m})$ for some $\mathbb{Q}(\sqrt{m}) \in \mathfrak{R}_{K}$, we can therefore determine their exact values, based on the numerical values of $G_{p q}$ and $G_{p / q}$, in a finite number of steps. This will in turn lead to exact values of $G_{p q}$.

Remark. Except for $K=\mathbb{Q}(\sqrt{-217})$ and $\mathbb{Q}(\sqrt{-553})$, in all of our calculations, $\left|\Re_{K}\right|=1$.

We illustrate our computations with two examples.

Before we proceed with the examples, we let $u:=G_{p q} G_{p / q}, v:=G_{p q} / G_{p / q}$, $U_{i}:=\left(u^{i}+u^{-i}\right)^{2}$, and $V_{j}:=\left(v^{j}+v^{-j}\right)^{2}$.

Example 1. Let $p=5$ and $q=13$. In this case, $\gamma=4$. By Theorem 7.3, $\alpha_{5,13}$ and $\beta_{5,13}$ are real quadratic algebraic integers. Since the primes 2, 5, and 13 are not inert in $\mathbb{Q}(\sqrt{65})$, we deduce that they are in $\mathbb{Q}(\sqrt{65})$.

Now, evaluating $u$ and $v$ using the product representation of $G_{n}$ (see (1.2)), we find that $\alpha_{5,13}=81.311288 \ldots$ and $\beta_{5,13}=57.186772 \ldots$. We know that these numbers are of the form $a+b \sqrt{65}$, and, by Theorem 7.4 , we conclude that

$$
\alpha_{5,13}=\frac{41+5 \sqrt{65}}{2} \quad \text { and } \quad \beta_{5,13}=\frac{33+3 \sqrt{65}}{2} .
$$

Therefore,

$$
U_{2}=\frac{45+5 \sqrt{65}}{2} \quad \text { and } \quad V_{2}=\frac{37+3 \sqrt{65}}{2}
$$


which implies that

$$
U_{1}=\frac{\sqrt{65}+9}{2} \quad \text { and } \quad V_{1}=\frac{\sqrt{65}+7}{2} .
$$

This further implies that

$$
u=\sqrt{\frac{\sqrt{65}+9}{8}}+\sqrt{\frac{\sqrt{65}+1}{8}} \quad \text { and } \quad v=\sqrt{\frac{\sqrt{65}-1}{8}}+\sqrt{\frac{\sqrt{65}+7}{8}} .
$$

Hence,

$$
G_{65}=\left(\sqrt{\frac{\sqrt{65}+9}{8}}+\sqrt{\frac{\sqrt{65}+1}{8}}\right)^{1 / 2}\left(\sqrt{\frac{\sqrt{65}+7}{8}}+\sqrt{\frac{\sqrt{65}-1}{8}}\right)^{1 / 2}
$$

and

$$
G_{13 / 5}=\left(\sqrt{\frac{\sqrt{65}+9}{8}}+\sqrt{\frac{\sqrt{65}+1}{8}}\right)^{1 / 2}\left(\sqrt{\frac{\sqrt{65}+7}{8}}-\sqrt{\frac{\sqrt{65}-1}{8}}\right)^{1 / 2} .
$$

Example 2. Let $p=3$ and $q=23$. In this case, $\gamma=12$. Using the numerical values of $u$ and $v$ and Theorems 7.3 and 7.4, we find that

$$
U_{6}=281344+162432 \sqrt{3} \quad \text { and } \quad V_{6}=2992+1728 \sqrt{3} .
$$

The first equality implies that

$$
u^{6}+u^{-6}=8(47+27 \sqrt{3}) .
$$

Since

$$
\begin{aligned}
\left(u^{2}+u^{-2}\right)^{3}-3\left(u^{2}+u^{-2}\right) & =u^{6}+u^{-6} \\
& =8(47+27 \sqrt{3}) \\
& =(4+3 \sqrt{3})^{3}-3(4+3 \sqrt{3}),
\end{aligned}
$$

we conclude that

$$
u^{2}+u^{-2}=4+3 \sqrt{3} \quad \text { and } \quad U_{1}=6+3 \sqrt{3} .
$$

Collecting our results and simplifying, we deduce that

$$
G_{69}=(\sqrt{748+432 \sqrt{3}}+\sqrt{747+432 \sqrt{3}})^{1 / 12}\left(\sqrt{\frac{6+3 \sqrt{3}}{4}}+\sqrt{\frac{2+3 \sqrt{3}}{4}}\right)^{1 / 2} .
$$

The following table summarizes our further calculations. The values for $G_{697}$ and $G_{793}$ are new. 


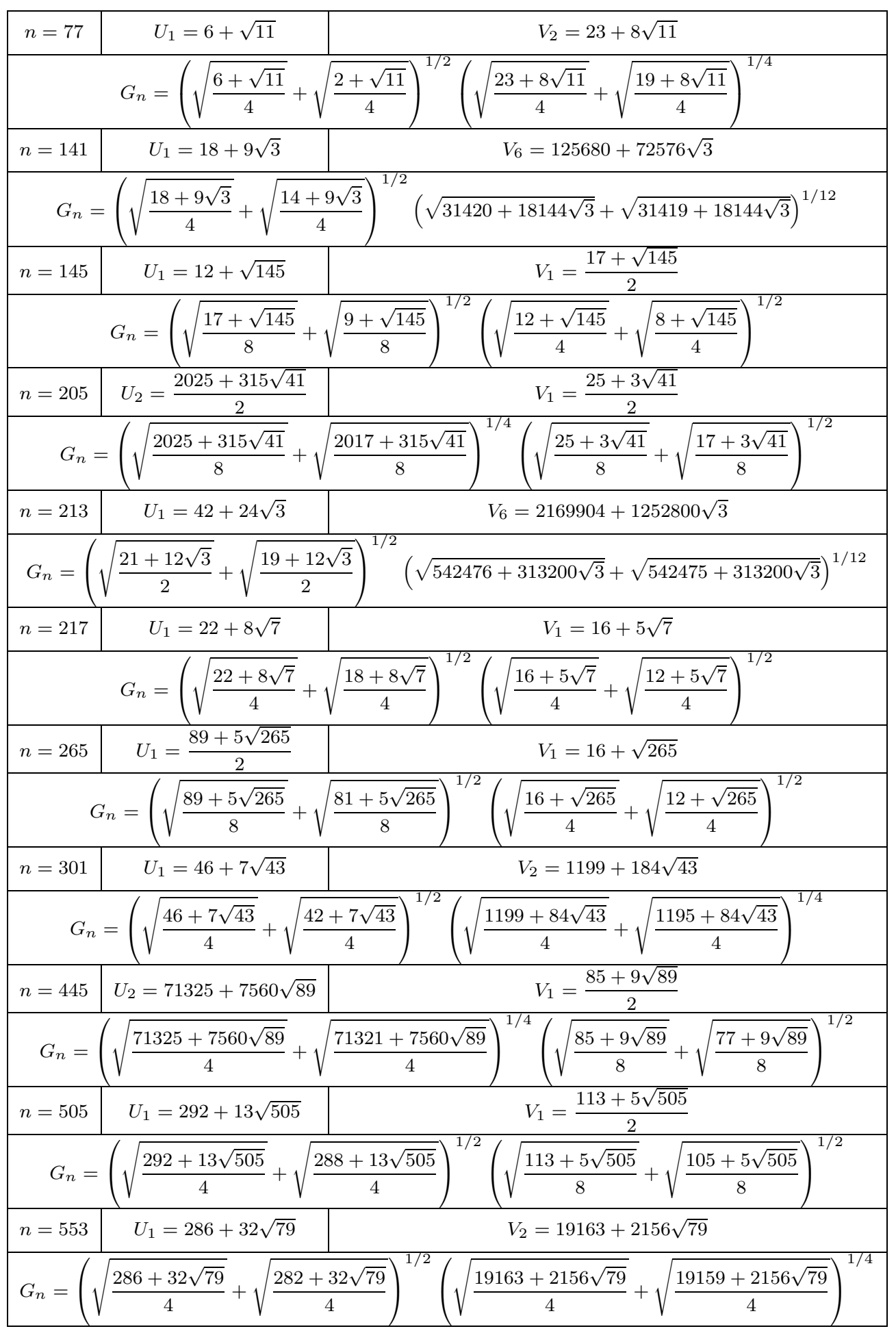




\begin{tabular}{|l|l|c|}
\hline$n=697$ & $U_{1}=\frac{769+29 \sqrt{697}}{2}$ & $V_{1}=\frac{661+25 \sqrt{697}}{2}$ \\
\hline$G_{n}=\left(\sqrt{\frac{769+29 \sqrt{697}}{8}}+\sqrt{\frac{761+29 \sqrt{697}}{8}}\right)^{1 / 2}\left(\sqrt{\frac{661+25 \sqrt{697}}{8}}+\sqrt{\frac{653+25 \sqrt{697}}{8}}\right)^{1 / 2}$ \\
\hline$n=793$ & $U_{1}=704+25 \sqrt{793}$ & $V_{1}=452+16 \sqrt{793}$ \\
\hline$G_{n}=\left(\sqrt{\frac{704+25 \sqrt{793}}{4}}+\sqrt{\frac{700+25 \sqrt{793}}{4}}\right)^{1 / 2}\left(\sqrt{\frac{452+16 \sqrt{793}}{4}}+\sqrt{\frac{448+16 \sqrt{793}}{4}}\right)^{1 / 2}$ \\
\hline
\end{tabular}

The authors are grateful to Jerry Keiper, Adam Strzebonski, and Michael Trott of Wolfram Research for help in denesting two equations with radicals. We also thank Steve Ullom and David Cox for their valuable suggestions and comments on the material in Section 7.

While this paper was being written, Jerry Keiper died tragically at the age of 41. Jerry was a very unselfish, unassuming talented mathematician and computer scientist. I frequently sought his advice on computational matters, and when I asked him a question about the denesting of two equations for this paper, he and his colleagues e-mailed me the denestings later on the same day. This was typical of Jerry; he was never too busy to help others, whether they be mathematicians or people living in Haiti or Nigeria, where he taught for three years. (BCB)

\section{REFERENCES}

1. B. C. Berndt, Ramanujan's Notebooks, Part III, Springer-Verlag, New York, 1991. MR 92j:01069

2. B. C. Berndt, Ramanujan's Notebooks, Part IV, Springer-Verlag, New York, 1994. MR 95e: 11028

3. B. C. Berndt and H. H. Chan, Some values for the Rogers-Ramanujan continued fraction, Canad. J. Math. 47 (1995), 897-914. CMP 96:01

4. B. C. Berndt, H. H. Chan, and L.-C. Zhang, Ramanujan's class invariants and cubic continued fraction, Acta Arith. 73 (1995), 67-85. CMP 96:03

5. B. J. Birch, Weber's class invariants, Mathematika 16 (1969), 283-294. MR 41:6816

6. Z. I. Borevich and I. R. Shafarevich, Number Theory, Academic Press, New York, 1966. MR 33:4001

7. J. M. and P. B. Borwein, Pi and the AGM, Wiley, New York, 1987. MR 89a:11134

8. D. A. Buell, Binary Quadratic Forms, Classical Theory and Modern Computations, SpringerVerlag, New York, 1989. MR 92b:11021

9. H. H. Chan and S. S. Huang, On the Ramanujan-Göllnitz-Gordon continued fraction, The Ramanujan Journal 1 (1997), 75-90.

10. H. Cohen, A Course in Computational Algebraic Number Theory, Springer-Verlag, Berlin, 1993. MR 94i: 11105

11. D. A. Cox, Primes of the Form $x^{2}+n y^{2}$, Wiley, New York, 1989. MR 90m:11016

12. M. Deuring, Die Klassenkörper der komplexes Multiplication, Enz. Math. Wiss., Band $I_{2}$, Heft 10, Teil II (Stuttgart) (1958). MR 29:4754

13. J. Janusz, Algebraic Number Fields, 2nd ed., Amer. Math. Soc., Providence, 1996.

14. R. Kortum and G. McNiel, A Table of Periodic Continued Fractions, Lockheed Aircraft Corporation, Sunnyvale, CA.

15. R. Mollin and L.-C. Zhang, Orders in quadratic fields II, Proc. Japan Acad., Ser. A 69 (1993), 368-371. MR 95d:11153

16. M. Pohst and H. Zassenhaus, Algorithmic Algebraic Number Theory, Cambridge University Press, Cambridge, 1989. MR 92b:11074

17. K. G. Ramanathan, Remarks on some series considered by Ramanujan, J. Indian Math. Soc. 46 (1982), 107-136. MR 88e:11077 
18. K. G. Ramanathan, On the Rogers-Ramanujan continued fraction, Proc. Indian Acad. Sci. (Math. Sci.) 93 (1984), 67-77. MR 87a:11012

19. K. G. Ramanathan, Ramanujan's continued fraction, Indian J. Pure Appl. Math. 16 (1985), 695-724. MR 87e:11015

20. K. G. Ramanathan, Some applications of Kronecker's limit formula, J. Indian Math. Soc. 52 (1987), 71-89. MR 90j:11112

21. K. G. Ramanathan, On some theorems stated by Ramanujan, Number Theory and Related Topics, Oxford University Press, Bombay, 1989, pp. 151-160.

22. S. Ramanujan, Modular equations and approximations to $\pi$, Quart. J. Math. (Oxford) 45 (1914), 350-372.

23. S. Ramanujan, Notebooks (2 volumes), Tata Institute of Fundamental Research, Bombay, 1957. MR 20:6340

24. S. Ramanujan, Collected Papers, Chelsea, New York, 1962.

25. J. Rotman, Group Theory, Springer-Verlag, New York, 1990. MR 91j:12009

26. R. Russell, On modular equations, Proc. London Math. Soc. 21 (1890), 351-395.

27. C. L. Siegel, Advanced Analytic Number Theory, Tata Institute of Fundamental Research, Bombay, 1980. MR 83m:10001

28. G. N. Watson, Theorems stated by Ramanujan (IX): two continued fractions, J. London Math. Soc. 4 (1929), 231-237.

29. G. N. Watson, Theorems stated by Ramanujan (XIV): a singular modulus, J. London Math. Soc. 6 (1931), 126-132.

30. G. N. Watson, Some singular moduli (I), Quart. J. Math. 3 (1932), 81-98.

31. G. N. Watson, Some singular moduli (II), Quart. J. Math. 3 (1932), 189-212.

32. G. N. Watson, Singular moduli (3), Proc. London Math. Soc. 40 (1936), 83-142.

33. G. N. Watson, Singular moduli (4), Acta Arith. 1 (1936), 284-323.

34. G. N. Watson, Singular moduli (5), Proc. London Math. Soc. 42 (1937), 377-397.

35. G. N. Watson, Singular moduli (6), Proc. London Math. Soc. 42 (1937), 398-409.

36. H. Weber, Lehrbuch der Algebra, dritter Band, Chelsea, New York, 1961.

Department of Mathematics, 1409 West Green Street, University of Illinois, UrBANA, ILLINOIS 61801

E-mail address: berndt@math.uiuc.edu

School of Mathematics, Institute for Advanced Study, Princeton, New Jersey 08540

Current address: Department of Mathematics, National Chung Cheng University, Minhsiung, Chiyai 621, Taiwan, R.O.C.

E-mail address: hhchan@mthmp.math.ccu.edu.tw

Department of Mathematics, Southwest Missouri State University, Springfield, MisSOURI 65804

E-mail address: liz917f@cnas.smsu.edu 